\title{
Synchronous nonmonotonic changes in functional connectivity and white matter integrity in a rat model of sporadic Alzheimer's disease
}

\author{
Catarina Tristão Pereira a,b,\#, Yujian Diao a,c,\#, Ting Yin ${ }^{\mathrm{a}}$, Analina R da Silva ${ }^{\mathrm{a}}$, Bernard Lanz ${ }^{\mathrm{c}}$, \\ Katarzyna Pierzchala ${ }^{a}$, Carole Poitry-Yamate ${ }^{a}$, Ileana O Jelescu ${ }^{\text {a,* }}$ \\ ${ }^{a}$ Centre d'Imagerie Biomédicale, EPFL, Station 6, Lausanne 1015, Switzerland \\ ${ }^{\mathrm{b}}$ Faculdade de Ciências da Universidade de Lisboa, Lisbon, Portugal \\ ${ }^{\mathrm{c}}$ Laboratoire d'Imagerie Fonctionnelle et Métabolique, EPFL, Lausanne, Switzerland
}

\section{A B S T R A C T}

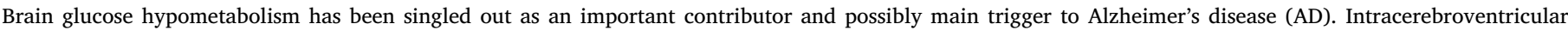

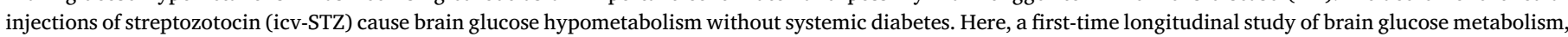

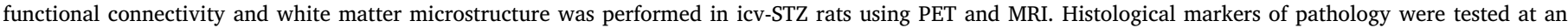

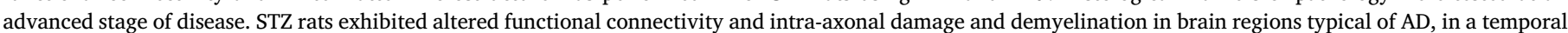

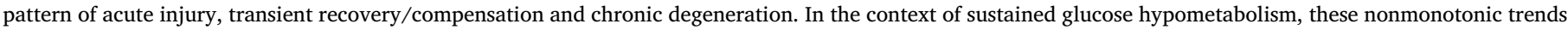

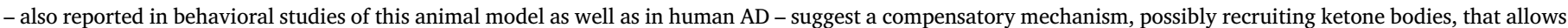

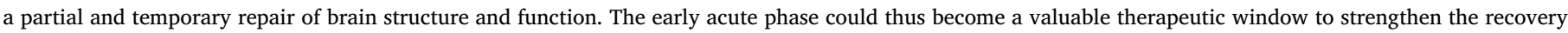

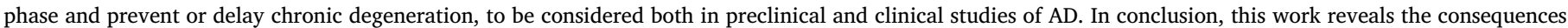

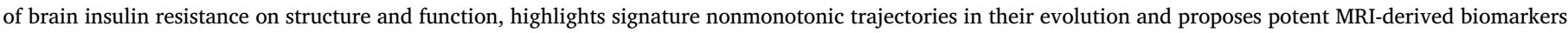
translatable to human $\mathrm{AD}$ and diabetic populations.

\section{Introduction}

Alzheimer's disease (AD) is the most common form of dementia and its incidence outburst already constitutes an economic and social burden. Clinically, $\mathrm{AD}$ is manifested by progressive memory loss and gradual decline in cognitive function, often culminating in premature death. However, pathological brain changes in AD begin to develop decades before the first symptoms (Sperling et al., 2011). The characterization of the temporal progression of these changes promises to provide an understanding of disease mechanisms, an effective disease staging and a window for therapeutic intervention.

In that regard, many studies have proposed the order in which relevant biomarkers occur in the progression of disease (Jack et al., 2013; Sperling et al., 2011). The pathological cascade of AD is characterized by initial deposition of amyloid- $\beta$ (A $\beta$ ) plaques in the extracellular space and intracellular neurofibrillary tangles of hyperphosphorylated tau (NFTs) (Dickerson, 2013; Shaw et al., 2009; Wang et al., 2013). In addition, neurodegeneration manifests as reduced brain glucose metabolism (Edison et al., 2007), impaired synaptic activity (Shankar and Walsh, 2009) and neuronal loss (Attwell and Laughlin, 2001; Zarow et al., 2005). Gross cerebral atrophy (Gispert et al., 2015) and white matter degeneration (Chang et al., 2015; Choo et al.,

\footnotetext{
* Corresponding author.

E-mail address: ileana.jelescu@epfl.ch (I.O. Jelescu).

\# These authors contributed equally to the work
}

2010; Dong et al., 2020; Jelescu et al., 2018) have been considered relevant biomarkers as well, along with reduced functional connectivity (Binnewijzend et al., 2012; Franzmeier et al., 2019). Nevertheless, the spatio-temporal relationship between $\mathrm{AD}$ hallmarks remains unclear.

Meanwhile, hypometabolism of glucose in the brain is taking the center stage as a key player in the onset of $\mathrm{AD}$ (Kuehn, 2020), while the amyloid cascade hypothesis is being questioned (Kametani and Hasegawa, 2018). Impaired brain insulin function has been reported to trigger AD pathology (Correia et al., 2011; Hölscher, 2019), while less robust glycolysis has been shown in animals at increased risk for developing dementia (Wu et al., 2018). Moreover, metabolic decline has been linked to impaired glucose transport and neuroinflammation (An et al., 2018; Balducci and Forloni, 2018). As it becomes wellestablished that diabetes makes the brain more susceptible to the aging process (Gudala et al., 2013), AD pathology has been suggested to be a brain-specific type-III diabetes (de la Monte and Wands, 2008).

Animal models allow studying each of the contributors to the cascade individually and obtaining comprehensive longitudinal data across their lifespan, which is particularly important for untangling direct effects of contributors and their interactions. Much effort has been put into the development and studies of transgenic mouse models of amyloidosis and tauopathy, which revealed for instance that - in mice - mutations causing amyloidosis alone do not produce NFTs, and tauopathy alone results in a fronto-temporal dementia pattern rather than AD (Buxbaum, 2009). 
The validity of transgenic animals is also limited in that they are not representative of the sporadic form of the disease, which is the predominant one in humans (King, 2018).

Numerous non-transgenic rat models have been proposed to replicate the $\mathrm{AD}$ phenotype, including models based on the ventricular infusion of the amyloid peptide (Sanganahalli et al., 2013); chemically-induced cholinergic-dysfunction models (Karthick et al., 2016); senescence-accelerated OXYS rats induced by a galactose-rich diet (Stefanova et al., 2015), and others (see (Benedikz et al., 2009) for a review). With the importance of brain glucose metabolism in AD being increasingly recognized, animal models of brain insulin resistance have been developed by an intracerebroventricular (icv) injection of streptozotocin (STZ). Intravenous injection of STZ is a very well-established model for type I diabetes (Ganda et al., 1976; Junod et al., 1969). Even though its mechanism is not fully elucidated, when delivered exclusively to the brain via the ventricles, this diabetogenic substance is thought to disrupt the brain insulin receptor system, thereby reducing glycolysis in the hippocampus and parieto-temporal cortex, but without causing systemic diabetes (Nitsch and Hoyer, 1991; Plaschke et al., 2010).

Accordingly, icv-STZ rats and monkeys have been shown to develop features typical of AD including memory impairment, NFT changes and extracellular accumulation of $\mathrm{A} \beta$ in the neocortex and hippocampus, thinning of the parietal cortex and corpus callosum (Knezovic et al., 2015), neuronal loss, astrocytosis and microgliosis in the corpus callosum and septum (Kraska et al., 2012), axonal damage and demyelination in the hippocampus and fimbria (Shoham et al., 2003) and decreased glucose uptake (Heo et al., 2011). Along with disruption of brain glucose metabolism, STZ induces oxidative stress by impairment of insulin signaling (Lester-Coll et al., 2006; Shoham et al., 2003).

In terms of STZ kinetics, previous studies have highlighted changes as early as one week and up to 39 weeks post-injection. Neurodegenerative lesions associated with inflammation have been reported in STZ rats at 1 and 13 weeks post-injection (Kraska et al., 2012). Tau and A $\beta$ accumulation as well as cognitive deficits have been reported in STZ rats at 4, 13, 26 and 39 weeks post-injection (Knezovic et al., 2015). Finally, a FDG-PET study has shown glucose metabolism alterations in STZ monkeys at 6 and 12 weeks post-injection (Heo et al., 2011). The icv-STZ model has clearly demonstrated its potential to reproduce sporadic AD by the resemblance with the behavioral, neurochemical and structural changes found in the human AD brain. However, beyond anatomical data, this animal model has never been assessed with MRI, which could potentially offer significant insight into neurodegenerative processes.

We hypothesize that MRI-derived metrics of microstructure and functional connectivity in icv-STZ rats should show the same biphasic pattern as reported in human AD (Dickerson et al., 2005; Dong et al., 2020; Montal et al., 2018), with early inflammation followed by remission due to compensatory mechanisms and chronic neurodegeneration. Furthermore, in icv-STZ rats we could determine how these changes relate to glucose hypometabolism.

Here we therefore performed a longitudinal study in the icv-STZ rat model to quantitatively characterize alterations in functional connectivity and in white matter microstructure using resting-state functional (rs-fMRI) and diffusion MRI, respectively, as well as changes in brain glucose uptake captured by ${ }^{18}$ FDG (fluorodeoxyglucose)-PET. The diffusion MRI analysis was augmented with an extensively validated biophysical model of white matter which gives access to specific metrics of microstructure such as axonal density or intra-axonal diffusivity (Jelescu et al., 2020; Jelescu and Budde, 2017; Jespersen et al., 2018). Histological markers of pathology such as amyloid and NFTs were also tested. This study highlights the dynamics of how brain insulin resistance affects structure and function in a spatio-temporal pattern. It experimentally supports the caveat that metabolism underlies structure and function. This work further serves the dual purpose of outlining pathological trajectories relevant for clinical studies and validating potent MRIderived biomarkers to track neurodegeneration in human AD and diabetes populations.

\section{Methods}

\subsection{Animals}

Animal experiments were approved by the Service for Veterinary Affairs of the canton of Vaud. Male Wistar rats (Charles River, l'Arbresle, France) weighing $218-259 \mathrm{~g}$ at baseline were assessed at three timepoints after the icv injection: PET and MRI data were collected at 2, 6 and 13 weeks following surgery (Fig. 1) and rats were sacrificed by perfusion fixation at 21 weeks for histological evaluation. Timepoints were chosen to be consistent with previous studies while also accommodating for constraints of repeated MRI and PET scanning related to anesthesia, cannulations, and scanner variability. Unfortunately, due to unforeseen technical issues with the PET scanner, the number of PET datasets was reduced at 2 weeks, and partially at 6 weeks. Later in the study, a major upgrade on the MRI system left the 13-week timepoint short of four MRI datasets.

\subsection{Intracerebroventricular injection}

Rats were anesthetized using isoflurane (4\% for induction and $2 \%$ for maintenance) and positioned in a stereotaxic frame (Kopf Model 900). The head was shaved and the skull was exposed with a midline incision. Two holes were burred into the skull, giving access to the two lateral ventricles at stereotaxic coordinates: $\pm 1.4 \mathrm{~mm}$ lateral, 0.9 posterior and $3.5 \mathrm{~mm}$ deep relative to the bregma. A single bilateral icv injection ( $2 \mu \mathrm{L}$ in each ventricle) of either $3 \mathrm{mg} / \mathrm{kg}$ STZ dissolved in citrate buffer (STZ group, $n=9$ ), or citrate buffer solution ( $0.05 \mathrm{M}, \mathrm{pH}=4.5$ ) (control group, CTL, $n=8$ ) was performed at a rate of $2 \mu \mathrm{L} / \mathrm{min}$ using Hamilton syringes, a double-syringe pump (TSE System) and catheters with $30 \mathrm{G}$ needles. The catheters were slowly removed $15 \mathrm{~min}$ after the end of the perfusion and the skin was sutured with silk sutures 3/0.1500 mg paracetamol was administered in $800 \mathrm{~mL}$ drinking water starting 2 days before and up to 3 days after the surgery and an antiseptic cream was applied locally for 5 days after surgery. Rats were allowed to recover for two weeks before the first scanning session. They were kept in pairs in each cage for the entire study, checked daily by the veterinary team, and had free access to food and water.

\subsection{FDG-PET data acquisition}

\subsubsection{Animal preparation}

Rats were initially anesthetized using isoflurane ( $2 \%$ for induction) in an oxygen/air mixture of $30 \% / 70 \%$ during tail vein cannulation for tracer delivery, and then isoflurane (1-2\%) in $100 \%$ oxygen via a nosecone once the animal was transferred and prone positioned on a temperature regulated PET scanner bed. Within the first minute of PET acquisition, a bolus of roughly $50 \mathrm{MBq}{ }^{18} \mathrm{~F}$-FDG (Advanced Accelerator Applications, Geneva, Switzerland) in 50-300 $\mu \mathrm{L}$ was manually injected through the tail vein and followed by a saline chase. Throughout the experiment, breathing rate and body temperature were monitored using a respiration pillow and a rectal thermometer, respectively. Body temperature was maintained around $(37 \pm 0.5)^{\circ} \mathrm{C}$. Pre- and post-scan glycemia were determined from blood at the tail vein cannulation site using a Bayer glucose meter.

\subsubsection{PET acquisition}

All PET experiments were performed on an avalanche photodiodebased LabPET-4 small-animal scanner (Gamma Medica-Ideas Inc.) as described in (Lanz et al., 2014). Briefly, data were collected in listmode and images between 30 and 50 minutes after injection were reconstructed using the maximum likelihood expectation maximization iterative reconstruction algorithm with a circular field of view (FOV) of $80 \mathrm{~mm}$. The reconstructed voxel size was $0.25 \times 0.25 \times 1.18 \mathrm{~mm}$. Steady-state radioactivity density images were then normalized for the 


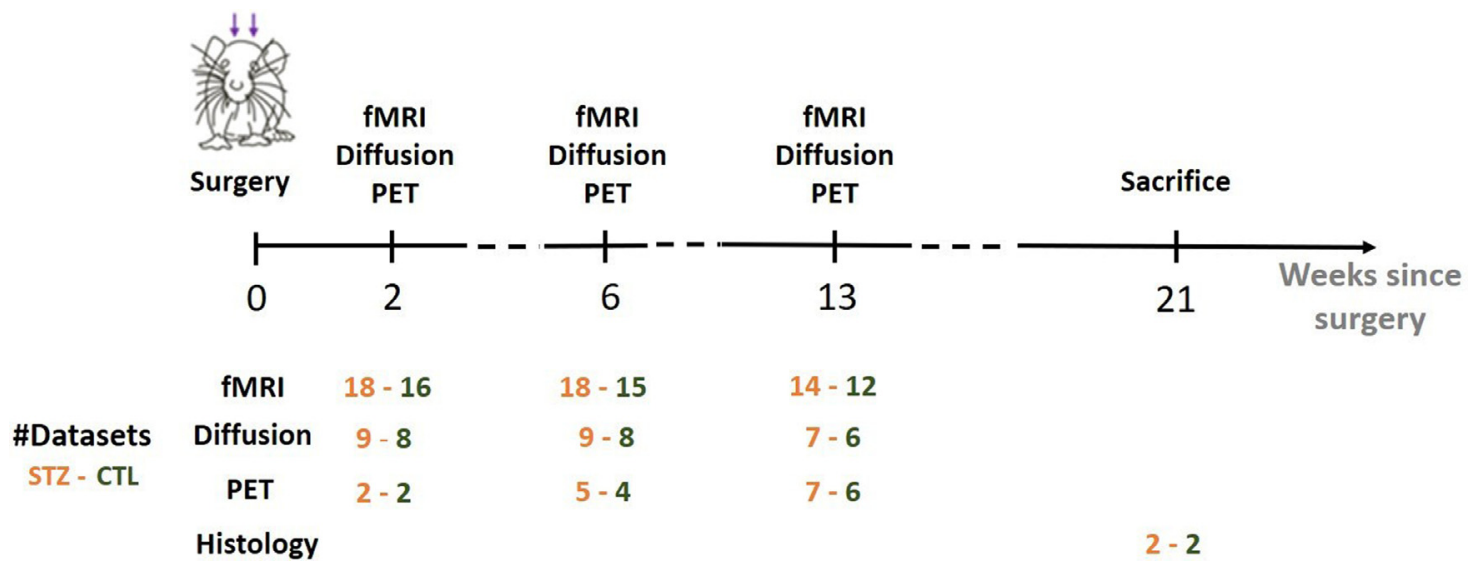

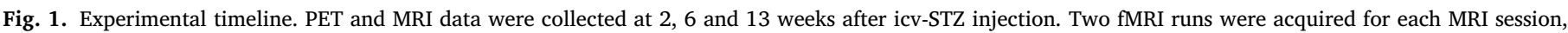
which explains the larger number of datasets. Histology was performed after animal sacrifice at 21 weeks.

effective injected FDG dose and the animal weight to generated Standardized Uptake Value (SUV) maps.

\subsection{MRI data acquisition}

\subsubsection{Animal preparation}

Animals were initially anesthetized using isoflurane (4\% for induction and $1-2 \%$ for maintenance in an oxygen/air mixture of 30\%/70\%) and positioned in a homemade MRI cradle equipped with a fixation system (bite bar and ear bars). A catheter was inserted subcutaneously on the back of the animal for later medetomidine delivery. One hour before starting the rs-fMRI acquisition, anesthesia was switched from isoflurane to medetomidine (Dorbene, Graeub, Switzerland), which preserves neural activity and vascular response better than isoflurane (Pawela et al., 2009; Weber et al., 2006), with an initial bolus of $0.1 \mathrm{mg} / \mathrm{kg}$ followed by a continuous perfusion of $0.1 \mathrm{mg} / \mathrm{kg} / \mathrm{h}$ (Reynaud et al., 2019). The commercial solution at $1 \mathrm{mg} / \mathrm{mL}$ was diluted to $0.033 \mathrm{mg} / \mathrm{mL}$. Throughout the experiment, the breathing rate and the body temperature were monitored similarly to the PET acquisition. The breathing rate under medetomidine was around $85 \mathrm{bpm}$. At the end of the scanning session, animals were woken up with an intramuscular injection of atipamezole (Alzane, Graeub, Switzerland) at $0.5 \mathrm{mg} / \mathrm{kg}$.

\subsubsection{MRI acquisition}

All MRI experiments were performed on a $14 \mathrm{~T}$ Varian system (Abingdon, UK) equipped with $400 \mathrm{mT} / \mathrm{m}$ gradients. An in-house built quadrature surface coil was used for transmission and reception.

Structural $\mathrm{T}_{2}$-weighted images were collected using a fast spin-echo sequence with the following parameters: TE/TR $=10.17 / 3000 \mathrm{~ms}$, echo train length: 4 , matrix size $=128 \times 128, \mathrm{FOV}=19.2 \times 19.2 \mathrm{~mm}^{2}$, voxel size $=0.15 \times 0.15 \mathrm{~mm}^{2}, 30$ coronal 0.5 - $\mathrm{mm}$ slices, scan time $=10 \mathrm{~min}$ utes.

Diffusion-weighted data were acquired using a semi-adiabatic pulsed-gradient spin-echo (PGSE) segmented echo-planar-imaging (EPI) sequence (van de Looij et al., 2011), with the following protocol: 4 $b=0$ images and 3 shells at $b=0.8 / 1.3 / 2.0 \mathrm{~ms} / \mu \mathrm{m}^{2}$, with 12,16 and 30 directions, respectively; $\delta / \Delta=4 / 27 \mathrm{~ms}$; TE/TR $=48 / 2500 \mathrm{~ms}$; 4 shots; matrix size $=128 \times 64$, FOV $=23 \times 17 \mathrm{~mm}^{2}$, voxel size $=0.18 \times 0.27 \mathrm{~mm}^{2}, 9$ coronal $1-\mathrm{mm}$ slices, 4 repetitions, scan time $=1$ hour.

Rs-fMRI data were acquired using a two-shot gradient-echo EPI sequence as follows: $\mathrm{TE} / \mathrm{TR}=10 / 800 \mathrm{~ms}, \mathrm{TR}_{\mathrm{vol}}=1.6 \mathrm{~s}$, matrix size $=64 \times 64$, FOV $=23 \times 23 \mathrm{~mm}^{2}$, voxel size $=0.36 \times 0.36 \mathrm{~mm}^{2}$ and 8 coronal 1.12-mm slices, 370 repetitions, scan time $=10$ minutes. Two fMRI runs were acquired in each MRI session.

\subsection{Histology}

At 21 weeks after the icv injection, rats were perfusion fixed using a $4 \%$ formaldehyde solution and the brains were promptly removed and immersed in 4\% formaldehyde for two days, washed with PBS and stored at $4^{\circ} \mathrm{C}$ until they were embedded in paraffin and then cut on a sliding microtome into $8 \mu \mathrm{m}$ thick slices. Mounted tissue sections were dried at room temperature overnight. Two brains per group were processed. Four main brain regions were investigated, centered around A-P atlas coordinates relative to bregma: $+1 \mathrm{~mm}$ and $-0.4 \mathrm{~mm}$ (covering the cingulate, motor and primary somatosensory (S1) cortices and the caudate putamen, $-2.5 \mathrm{~mm}$ (covering the retrosplenial (RSC) and S1 cortices, hippocampus, thalamus and hypothalamus) and $-3.6 \mathrm{~mm}$ (covering the RSC, S1, auditory and parietal cortices, medial temporal lobe (MTL), hippocampus, thalamus and hypothalamus). Eight sections from each of these regions were stained with each of these contrasts: Congo red for $\mathrm{A} \beta$ plaques and Gallyas and Bielschowsky for NFTs.

\subsubsection{Amyloid accumulation}

Amyloid pathology was assessed with Congo Red staining (IHC WORLD, LLC). Slides were deparaffinized and rehydrated in distilled water, then incubated in Congo red working solution for 10 minutes and rinsed in distilled water. Finally, they were differentiated in alkaline alcohol solution, dehydrated through $95 \%$ and $100 \%$ alcohol then cleaned in xylene and mounted with resinous mounting medium.

\subsubsection{Neurofibrillary tangles}

Staging of tau protein pathology was first explored using the Gallyas Silver Stain method and the protocol described in https://www. protocolsonline.com/histology/dyes-and-stains/neurohistology/gallyassilver-stain/.

\subsubsection{Myelin staining}

Myeloarchitecture was evaluated using LFB staining. Slides were deparaffinized and rehydrated, and then incubated in LFB solution at $56^{\circ} \mathrm{C}$ overnight in the dark. The excess stain was rinsed with $95 \%$ ethyl alcohol followed by distilled water. Then slides were differentiated in a lithium carbonate solution for $30 \mathrm{~s}$, followed by $70 \%$ ethyl alcohol for $30 \mathrm{~s}$, rinsed in distilled water, dehydrated in $100 \%$ alcohol, cleaned in xylene and finally mounted with resinous mounting medium.

\subsubsection{Imaging}

Stained slices were then imaged and digitized using a MEIJI TECHNO TC5600 Inverted Biological Microscope and were visually inspected for relevant staining features in STZ rats in comparison to the age-matched controls. Images were collected using the $\times 5, \times 20$ and $\times 50$ objectives at the same resolution. 


\subsection{Image processing}

\subsubsection{FDG uptake}

FDG-PET steady-state SUV maps were registered to their corresponding $\mathrm{T}_{2}$-weighted anatomical $\mathrm{MR}$ images with crosscorrelation using ANTs (Avants et al., 2011, 2008), which was in turn registered to the Waxholm Space Atlas of the rat brain (https://www.nitrc.org/projects/whs-sd-atlas) using linear and nonlinear registration (Avants et al., 2011) and 26 ROIs were automatically segmented. SUV images were normalized by the mean SUV over the brain to obtain SUVr maps corrected for inter-rat experimental variability (Heo et al., 2011).

\subsubsection{Resting-state functional connectivity}

The rs-fMRI pre-processing pipeline is described in detail in (Diao et al., 2020). Briefly, images were skull-stripped, denoised (in the sense of thermal noise reduction) using a novel Marchenko Pastur Principle Component Analysis (MP-PCA) approach ${ }^{2}$ (Ades-Aron et al., 2018; Does et al., 2019; Veraart et al., 2016), corrected for EPI-related distortions using FSL's topup (Smith et al., 2004), slice-timing corrected and spatially smoothed with a $0.36 \times 0.36 \times 1 \mathrm{~mm}^{3}$ full width half maximum (FWHM) kernel (Friston et al., 2007). Independent component analysis (ICA) was run on the fMRI timecourses using FSL's MELODIC (Beckmann and Smith, 2004), with a high-pass filter $(f>0.01 \mathrm{~Hz})$ and 40 components. All ICA decompositions were run through a dedicated rat FMRIB's ICA-based Xnoiseifier (FIX) classifier (Diao et al., 2020), as previously described for humans and mice (Griffanti et al., 2017; Zerbi et al., 2015) and artefactual independent components attributable to physiological noise were automatically removed from the signal. Corrected fMRI images were registered to the anatomical image and then to the Waxholm Space Atlas using linear and non-linear registration in ANTs (Avants et al., 2008) and 28 atlas-defined ROIs (14 per hemisphere) were automatically segmented. Lastly, mean fMRI timecourses were extracted for each ROI based on "FIX-cleaned" data and individual ROI-to-ROI functional connectivity matrices were computed by calculating partial correlation between timecourses covarying for the global signal.

\subsubsection{Diffusion MRI and white matter microstructure}

Pre-processing diffusion data included MP-PCA denoising (Veraart et al., 2016) followed by Gibbs-ringing correction ${ }^{3}$ (Kellner et al., 2016) and correction for EPI-related distortions, eddy currents and motion using FSL's eddy (Andersson and Sotiropoulos, 2016). The diffusion and kurtosis tensors were estimated using a weighted linear least squares algorithm ${ }^{4}$ (Jensen et al., 2005; Veraart et al., 2013) and typical DTI and DKI-derived metrics were calculated: fractional anisotropy (FA), mean/axial/radial diffusivity $(\mathrm{MD} / \mathrm{AxD} / \mathrm{RD})$ and mean/axial/radial kurtosis (MK/AK/RK). To overcome the lack of specificity inherent to empirical representations such as DTI and DKI, the biophysical WMTI model was computed in white matter voxels using a Watson distribution of axon orientations (Fieremans et al., 2011; Jespersen et al., 2018). The following microstructure parameters were extracted from the model: the intraaxonal water fraction $f$, the parallel intra-axonal diffusivity $D_{\text {a }}$, the parallel $D_{\mathrm{e}, \|}$ and perpendicular $D_{\mathrm{e}, \perp}$ extra-axonal diffusivities and the axons' orientation distribution $c_{2}$ (Fig. 2). This model has two mathematical solutions (Fieremans et al., 2011; Jelescu et al., 2016a; Novikov et al., 2018), of which the $D_{\mathrm{a}}>D_{\mathrm{e}, \|}$ solution was retained, according to recent evidence (Dhital et al., 2019; Jespersen et al., 2018; Kunz et al., 2018; Veraart et al., 2018). FA maps were registered to an FA template in the Waxholm Space using linear and non-linear registration in FSL (Jenkinson et al., 2002) and the corpus callosum, cingulum

\footnotetext{
2 https://github.com/NYU-DiffusionMRI/mppca_denoise

3 https://bitbucket.org/reisert/unring/

4 https://github.com/NYU-DiffusionMRI/DESIGNER
}

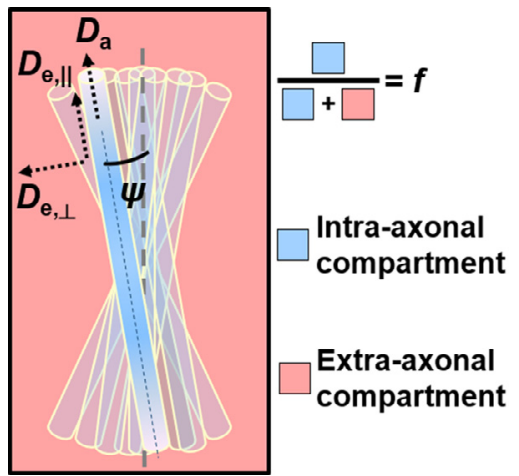

Fig. 2. Schematic of the WMTI-Watson biophysical model (Jelescu and Budde, 2017; Jespersen et al., 2018). The diffusion signal is described in terms of two non-exchanging compartments, the intra and extra-axonal spaces. Here, the axons are modelled as long narrow cylinders. The intra-axonal space is described by a volume fraction of water $f$ and by the parallel intra-axonal diffusivity $D_{\mathrm{a}}$. The perpendicular intra-axonal diffusivity is negligible at the relevant diffusion times and weightings, such that axons are considered to have radius equal to zero (effectively sticks). The bundle of axons is embedded in the extra-axonal space, characterized by its parallel $D_{\mathrm{e}, \|}$ and perpendicular extra-axonal diffusivities $D_{\mathrm{e}, \perp}$. The axons' orientations are modeled by a Watson distribution, which is characterized by $\left\langle(\cos \psi)^{2}\right\rangle \equiv c_{2}$. (For interpretation of the references to color in this figure legend, the reader is referred to the online version of this article.)

and fimbria of the hippocampus were automatically segmented. This approach enabled a more accurate segmentation of the white matter tracts, that otherwise tend to be misaligned due to enlargement of ventricles in STZ rats. Diffusion metrics were averaged over white matter ROIs.

\subsection{Statistical analysis}

The number of datasets available per group and timepoint in each type of analysis (PET, rs-fMRI and diffusion MRI) are reported in Fig. 1.

FDG uptake: Regional differences in SUVr between STZ and CTL groups were evaluated at each timepoint (except at 2 weeks due to the small sample size) using one-tailed Mann-Whitney $U$ test (STZ < CTL), at significance level of $\alpha=0.05$. At 2 weeks, regional mean and standard deviation in each group were considered instead. The effect size of group differences was calculated using Hedge's $g$ corrected for small sample size at 6 and 13 weeks post-injection.

Resting-state functional connectivity: The significance of group differences was tested using non-parametric permutation tests $(N=5000)$ with the Network-Based Statistics (NBS) Toolbox, with statistical significance retained at $p=0.05$ after family-wise error rate (FWER) correction.

White matter microstructure: Average diffusion-derived metrics in each white matter ROI were compared between STZ and CTL groups at each timepoint using two-tailed Mann-Whitney $U$ test, at $\alpha=0.05$ significance. Longitudinal changes within each group were assessed by oneway ANOVA and Tukey-Cramer correction, at $\alpha=0.05$ significance. The effect size of group differences was calculated using corrected Hedge's $g$.

Weight: Rats' weight was compared between STZ and CTL groups at each timepoint using two-tailed Mann-Whitney $U$ test, at significance level of $\alpha=0.05$. In this analysis, we included 9/8 STZ/CTL rats at baseline, 2 and 6 weeks after injection and 8/8 STZ/CTL rats at 13 weeks.

\section{Results}

Nine STZ rats and eight CTL were included in the longitudinal study. The datasets available for each modality (diffusion MRI, resting-state fMRI, FDG-PET and histology) at each of the four timepoints - 2, 6, 13 and 21 weeks - are provided in Fig. 1. 


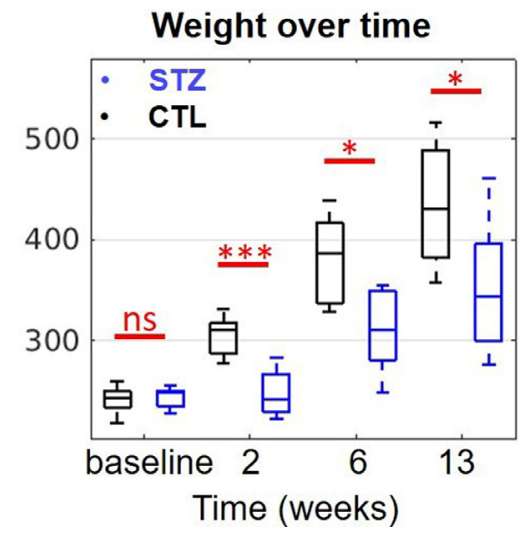

Fig. 3. Group differences in animal weight measured before icv-STZ injection (baseline) and at all timepoints. Boxes represent median, 25th and 75th percentiles. ns: non-significant, ${ }^{*}: p<0.05,{ }^{* * *}: p<0.001$ using the two-tailed Mann-Whitney $U$ test. 2 weeks: $p=0.0002,6$ weeks: $p=0.0152,13$ weeks: $p=$ 0.0499 . (For interpretation of the references to color in this figure legend, the reader is referred to the online version of this article.)

\subsection{Weight}

The weight of all animals was measured before the icv-STZ injection (baseline) and at all timepoints before each MRI or PET scanning session. There was no difference between groups at baseline, but STZ rats had a significantly lower weight than CTL rats at 2, 6 and 13 weeks after the injection (Fig. 3).

\subsection{FDG uptake}

Representative SUVr maps are provided as Supplementary Figure S1. Relative Standardized Uptake Value (SUVr) was reduced in STZ rats compared to CTL rats, with different spatial patterns over time (Fig. 4). At 2 weeks after injection, there were no differences between the STZ and the CTL groups in any of the regions of interest (ROIs) but the sample size was also very limited at that timepoint ( 2 animals per group). The most pronounced differences were found at 6 weeks after injection, involving several regions typically affected in AD. Lower SUVr was found in the anterior cingulate cortex (ACC) more significantly, but also in the retrosplenial cortex (RSC), the posterior parietal cortex (PPC), the motor, somatosensory, auditory and visual cortices. The medial temporal lobe (MTL) and the hippocampus (including subiculum) displayed a trend for lower SUVr with $p<0.1$. Later, STZ rats showed less significant group differences throughout the brain, with the MTL being affected at 13 weeks. The effect sizes were large, varying between 1.16 and 2.06 (Hedge's $g$ values corrected for small sample size). Supplementary Table $S 1$ collects all $p$-values and corrected Hedge's $g$ values for group differences at each timepoint.

\subsection{Resting-state functional connectivity}

Significant group differences in functional connectivity were found at 2, 6 and 13 weeks after injection (Fig. 5A). In Fig. 5B, graph networks highlight the group differences in nodal connections. At 2 weeks after injection, STZ rats displayed increased positive connectivity between the ACC and the RSC and decreased anti-correlation of the default mode network (DMN) (including the ACC, RSC, hippocampus and subiculum) to regions of the lateral cortical network (LCN) including S1 and the motor cortex, as compared to CTL rats. At 6 weeks, the main changes in STZ rats remained reduced anti-correlations between the DMN (RSC, PPC and hippocampus) and LCN (S1/S2) as well as striatum. STZ rats also displayed increased connectivity between the hippocampus and the thalamus and between the MTL and the visual cortex compared to CTL rats. At 13 weeks, there was a dramatic shift towards overall reduced functional connectivity of the DMN in STZ rats, with weaker positive correlations between ACC, PPC, RSC, MTL, hippocampus, subiculum and visual cortex. STZ rats also presented a mild anti-correlation between the hypothalamus and the ACC, whereas these regions were uncorrelated in CTL rats. Remarkably, STZ rats exhibited a nonmonotonic trend in functional connectivity alterations, moving from hyperconnectivity within the DMN and less efficient dissociation between the DMN and LCN, to hypoconnectivity within the DMN. This switch occurred between the 6 and 13-week timepoints.

\subsection{White matter microstructure}

Representative diffusion images ( $b=0$ and FA maps) are provided in Supplementary Figure S2. Metrics derived from the classical Diffusion Tensor Imaging (DTI) technique provided differences between groups, which were very marked in the corpus callosum and fimbria of the hippocampus and milder in the cingulum (Fig. 6). Compared to controls, STZ rats displayed lower fractional anisotropy (FA) in the corpus callosum at 2, 6 and 13 weeks after injection, in the fimbria at 2 and 13 weeks and in the cingulum at 13 weeks only. Metrics derived from Diffusion Kurtosis Imaging (DKI) - a clinically-feasible extension of DTI which also informs on tissue complexity - only captured group differences at 13 weeks after injection, when mean kurtosis (MK) was reduced in the STZ group in all ROIs. STZ rats also displayed lower axial (AK) and radial Kurtosis (RK) at 13 weeks in the corpus callosum and the fimbria and lower RK in the cingulum (not shown). The fimbria of the hippocampus was the white matter tract with the largest differences between STZ and CTL rats.

The "White Matter Tract Integrity (WMTI)-Watson" biophysical model provided a more specific characterization of white matter degeneration than DTI and DKI. In the corpus callosum, both axonal fraction $f$ and intra-axonal diffusivity $D_{a}$ were reduced in the STZ group at 2 weeks and 13 weeks (Fig. 7). In the fimbria, $f$ was lower in the STZ group at all timepoints, being more accentuated at 13 weeks, when $D_{a}$ was also lower. In the cingulum, reduced $f$ was found at 13 weeks. Other model metrics did not show significant changes between groups or over time.

All group differences were supported by very large effect sizes, with absolute corrected Hedge's $g$ values varying between 0.84 and 1.83 in tensors derived metrics and between 0.87 and 2.12 in WMTI-derived metrics.

Longitudinal differences consisted in an increase in FA in the cingulum in the CTL group between 6 and 13 weeks post-injection ( $p=$ 0.0348), while in the STZ group there was a significant decrease in AK in the corpus callosum between 6 and 13 weeks ( $p=0.0357$, not shown).

Group differences ( $p$-values) and effect sizes (corrected Hedge's $g$ ) at each timepoint for all tensor metrics and WMTI metrics are provided in Supplementary Table S2 and Table S3, respectively.

Overall, white matter integrity displayed a nonmonotonic trend with partial recovery at 6 weeks, most markedly in the fimbria but also in the corpus callosum. The 13-week timepoint appeared as one of the most widespread damage, with involvement of the cingulum bundle as well.

\subsection{Histology}

At 21 weeks post-injection, Congo red staining revealed amyloid accumulation, both intra and extra-cellularly, in the primary motor (M1), primary and secondary somatosensory (S1/S2, including the barrel field, trunk, dysgranular zone), agranular retrosplenial (RSA) and auditory (Au) cortices of STZ rats (Fig. 8). In the motor and somatosensory cortex, an extracellular diffused red staining in the neighborhood of positively stained intracellular $\mathrm{A} \beta$ was observed $(\mathrm{C}, \mathrm{F})$, which can be related to diffused premature $\mathrm{A} \beta$ plaques. No amyloid deposits were found in other regions, and notably not in hippocampus.

Gallyas staining revealed abnormal tau-protein in the pre-tangle status in the cingulate ( $\mathrm{Cg} 1)$, primary motor (M1) and somatosensory cor- 

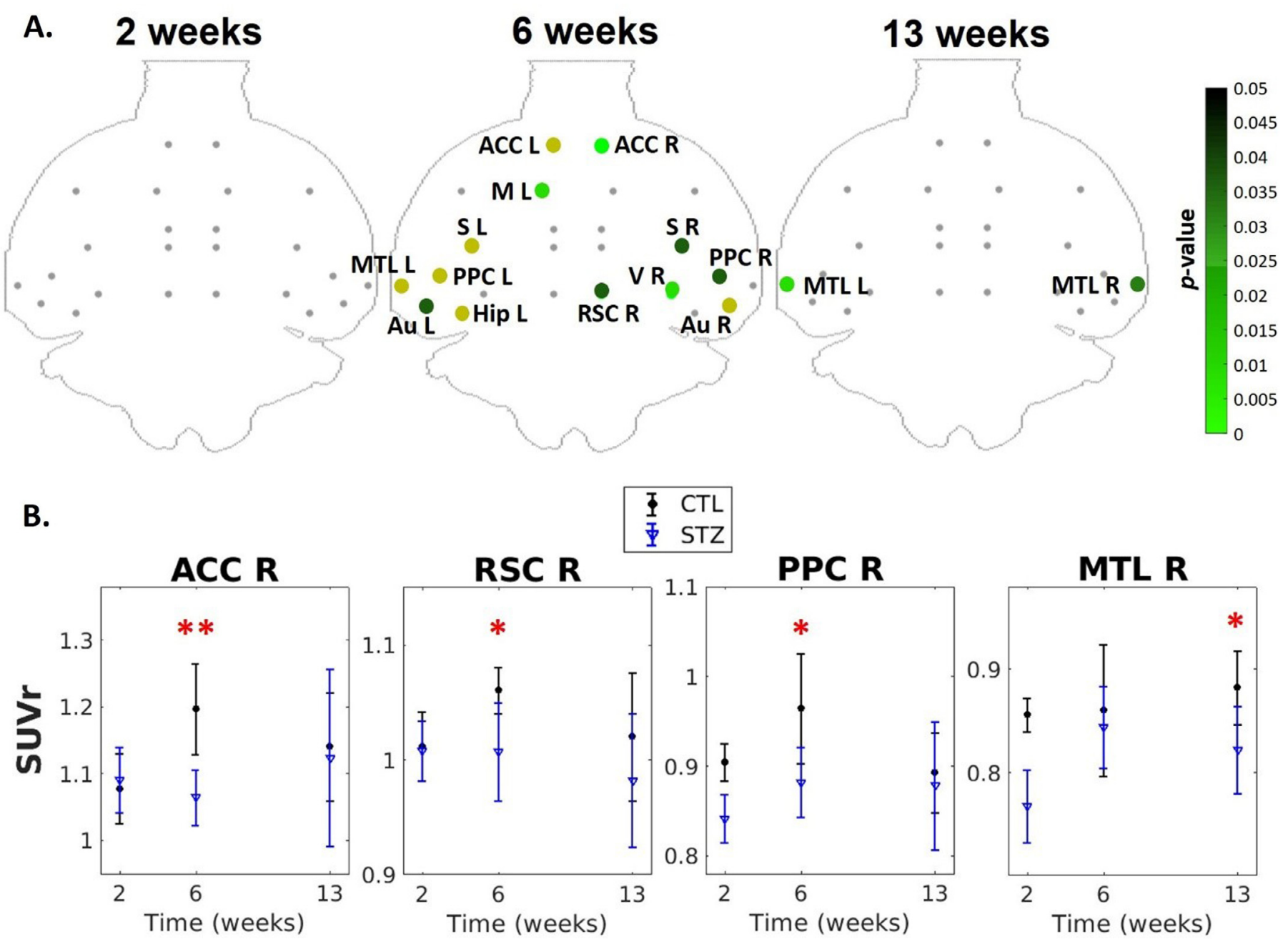

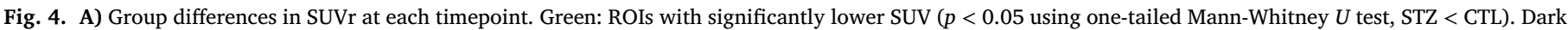

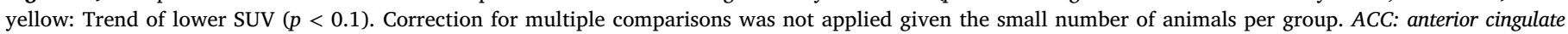

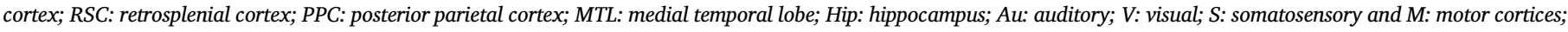

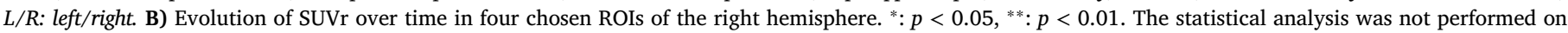

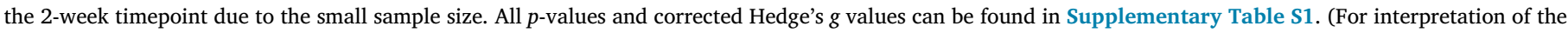
references to color in this figure legend, the reader is referred to the online version of this article.)

tices (S1) in both CTL and STZ rats (Fig. 9). STZ animals also exhibited a more advanced form of NFTs in S1. It should be noted that due to technical difficulties, Gallyas stain was compromised on the slices corresponding to more posterior brain regions ( $\mathrm{AP}<0 \mathrm{~mm}$ ), precluding the assessment of NFTs in posterior cortical areas and hippocampus.

Luxol fast blue (LFB) revealed macroscopic atrophy of brain structures, particularly the fimbria, and enlargement of the lateral ventricles in STZ rats. This feature was also apparent on in vivo MRI anatomical images (Fig. 10).

\section{Discussion and conclusions}

The icv-STZ animal model has reportedly shown alterations typical of $\mathrm{AD}$, reinforcing the idea that neurodegeneration may be primarily induced by brain insulin resistance. The effects of brain insulin resistance on the pathological cascade are however not fully understood, and the present study attempts to characterize them by means of MRIderived biomarkers for the first time. In this longitudinal assessment, FDG uptake, resting-state functional connectivity and white matter microstructure results agree in terms of spatio-temporal variations, and are consistent with our histological findings and with previous studies of cognitive decline and immunochemistry.

Functional connectivity in the CTL group was consistent with previous reports that midline regions of the DMN are anti-correlated with the sensorimotor system (Gozzi and Schwarz, 2016). In STZ animals, these anti-correlations between DMN and LCN were initially reduced (2 and 6 weeks) suggesting less efficient network dissociation and brain processing (Fox et al., 2005). Concomitantly, hyperconnectivity within the DMN was found in STZ rats at these early timepoints, broadly consistent with initial hyperconnectivity of the hippocampus in human studies of mild cognitive impairment (Dickerson et al., 2005). By 13 weeks however, STZ rats exhibited on the contrary reduced connectivity (or hypoconnectivity) in regions typically involved in $\mathrm{AD}$, also consistent with eventual memory impairment in this animal model and reduced functional connectivity in AD patients (Binnewijzend et al., 2012; Franzmeier et al., 2019). Changes in functional connectivity thus displayed a nonmonotonic pattern, with initial hyper-connectivity and impaired network dissociation, followed by later hypo-connectivity. The switch occurred between the 6 and 13-week timepoints.

White matter degeneration is best assessed when examining results from the DTI/DKI analysis and from the WMTI-Watson model jointly. 

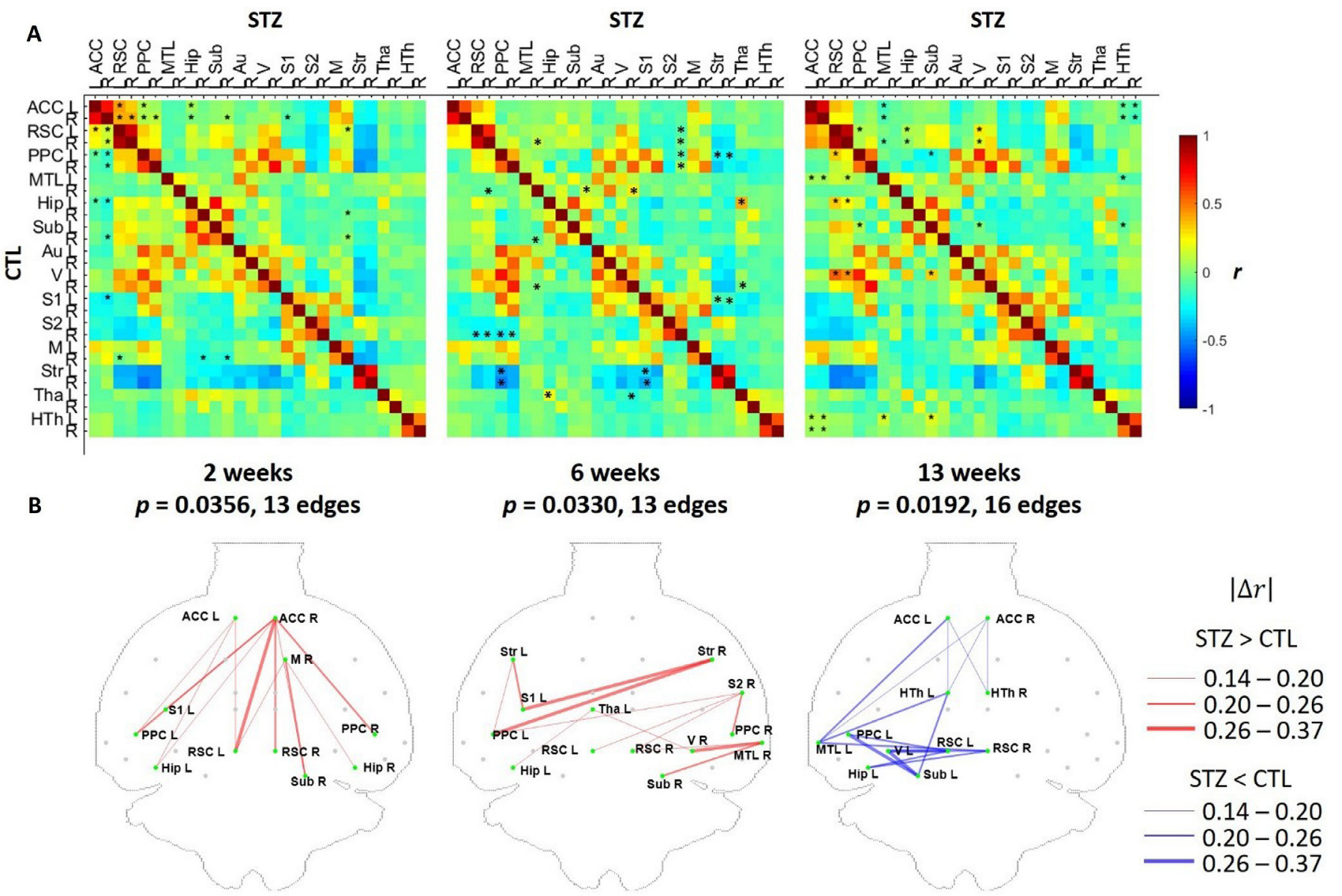

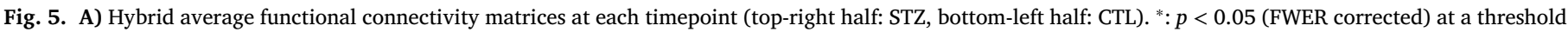

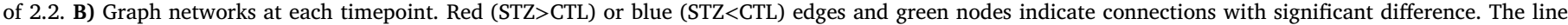

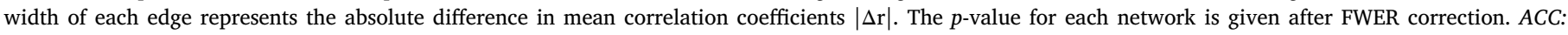

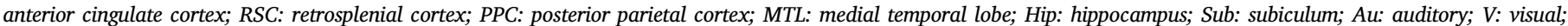

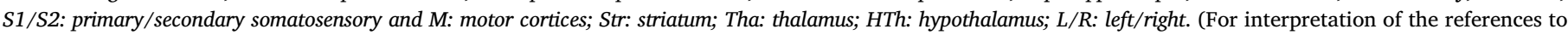
color in this figure legend, the reader is referred to the online version of this article.)

In the corpus callosum, reduced anisotropy was detected throughout the 2-13 week span, but subtle specific differences, in the form of reduced axonal fraction $f$ and intra-axonal diffusivity $D_{\text {a }}$ for example, were only found at 2 and 13 weeks, not at 6 weeks. Similarly, reduced anisotropy in the fimbria was detected at 2 and 13 weeks, while the WMTI-Watson model revealed reduced axonal fraction $f$ throughout the 2-13 week span, but with least pronounced differences at 6 weeks. Thus the 6week timepoint appears as one of partial recovery, before a marked degeneration at 13 weeks, with reduced anisotropy, mean kurtosis, axonal fraction and intra-axonal diffusivity in all three white matter bundles examined.

DTI and DKI metrics are indicative of a loss of tissue structure and complexity but do not provide specific information on the underlying changes. The characterization of white matter damage was further improved by biophysical modeling (Jelescu et al., 2020; Jelescu and Budde, 2017). Reduced intra-axonal diffusivity $D_{a}$ and axonal fraction $f$ were indicative of acute intra-axonal injury and demyelination at 2 weeks, followed by partial remission at 6 weeks and finally chronic injury and demyelination, potentially leading up to axonal loss at 13 weeks. While demyelination can lead to increased extra-axonal diffusivity perpendicularly to axons $\left(D_{e, \perp}\right)$, our previous work using electron microscopy validation has shown that the demyelination needs to be widespread for this parameter to be altered, while "patchy" demyelination will immediately be manifest as decreased axonal fraction $f$ (myelin is invisi- ble in diffusion MRI and lost myelin is replaced by extra-axonal space) (Jelescu et al., 2016b). The absence of changes in the other model parameters (water diffusivity in the extra-axonal space, or axons orientation dispersion) contributes to a specific picture of white matter microstructure alterations, with most damage being attributed to the axonal space, which is consistent with intra-cellular toxic action of STZ (Knezovic et al., 2015; Lester-Coll et al., 2006). Histological evidence of neuronal loss leading to macroscopic thinning of corpus callosum (Knezovic et al., 2015) and of axonal damage and demyelination of the fimbria (Shoham et al., 2003) have been previously reported in STZ rats and attributed to oxidative stress (Shoham et al., 2003). In humans, partial loss of myelin, axons and oligodendroglial cells have been observed histologically in early AD (Brun and Englund, 1986; Englund et al., 1988; Kobayashi et al., 2002; Roher et al., 2002; Svennerholm and Gottfries, 1994). Recent non-invasive MRI methods including diffusion MRI and relaxometry also provide evidence for demyelination in mild cognitive impairment and early AD (Bouhrara et al., 2018; Dong et al., 2020). Current theories further suggest a two-step demyelination process, with initial oligodendrocyte degeneration releasing iron, which promotes amyloid plaques, which in turn further destroy myelin until the neurodegenerative stage (Bartzokis, 2011, 2004).

Overall, changes in functional connectivity agreed with those in white matter microstructure both spatially and temporally. Remarkably, hypo-connectivity of cingulate cortex and hippocampus at 13 weeks 


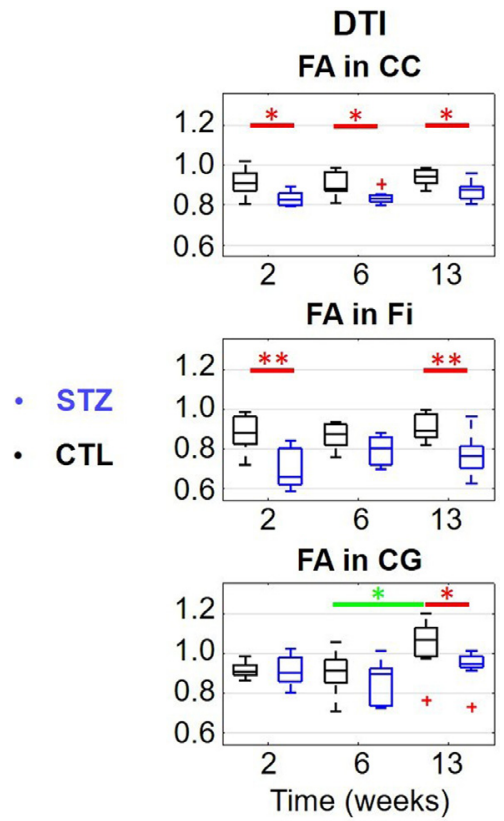

DKI
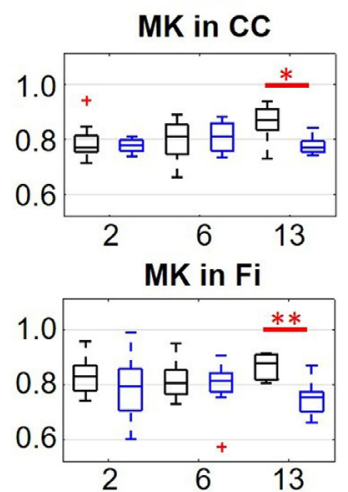

FA in CG

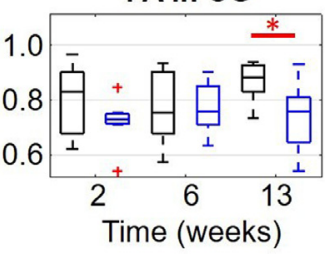

Fig. 6. DTI and DKI estimates in three white matter ROIs (top row: corpus callosum (CC), middle row: fimbria of the hippocampus (Fi) and bottom row: cingulum (CG)). FA: Fractional Anisotropy, MK: Mean Kurtosis. Two-tailed MannWhitney $U$ test for inter-group comparison (red bars) and one-way ANOVA with Tukey-Cramer correction for within-group comparison across time (green bars). *: $p<0.05,{ }^{* *}: p<0.01$. +: outlier values (but not excluded from the analysis). All $p$-values and corrected Hedge's $g$ values can be found in Supplementary Table S2. (For interpretation of the references to color in this figure legend, the reader is referred to the online version of this article.)

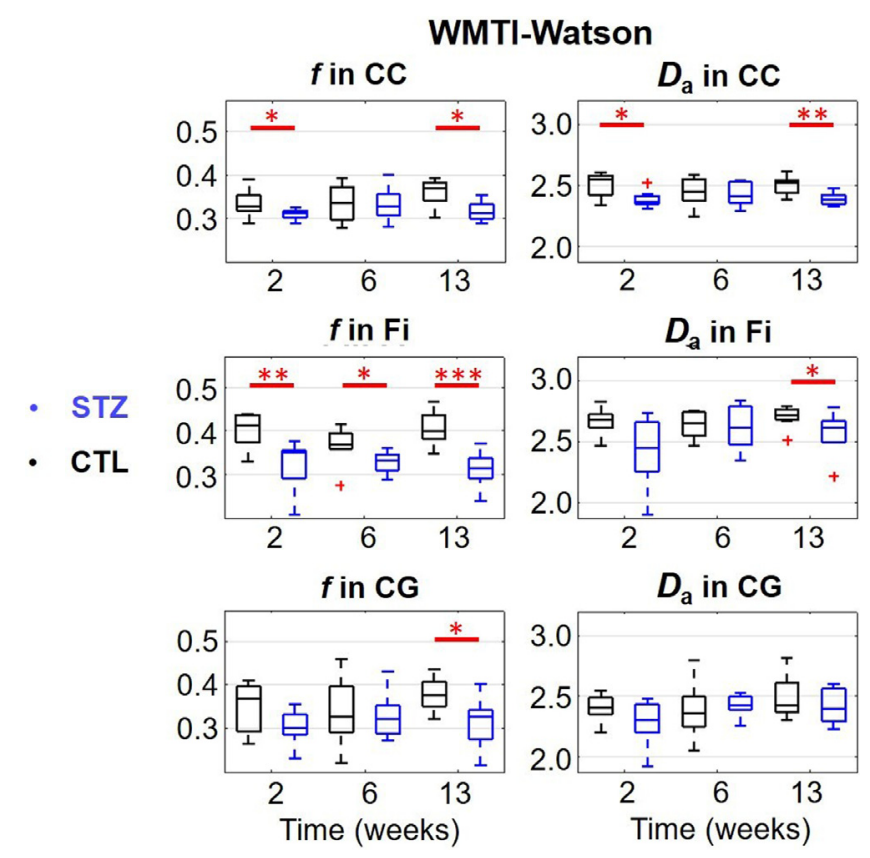

Fig. 7. WMTI-Watson model estimates in three white matter ROIs (top row: corpus callosum (CC), middle row: fimbria of the hippocampus (Fi) and bottom row: cingulum (CG)). $f$ : axonal water fraction, $D_{\mathrm{a}}$ : intra-axonal parallel diffusivity. Two-tailed Mann-Whitney $U$ test for inter-group comparison (red bars). ${ }^{*}: p<0.05,{ }^{* *}: p<0.01,{ }^{* * *}: p<0.001$. + : outlier values (but not excluded from the analysis). All $p$-values and corrected Hedge's $g$ values can be found in Supplementary Table S3. (For interpretation of the references to color in this figure legend, the reader is referred to the online version of this article.)

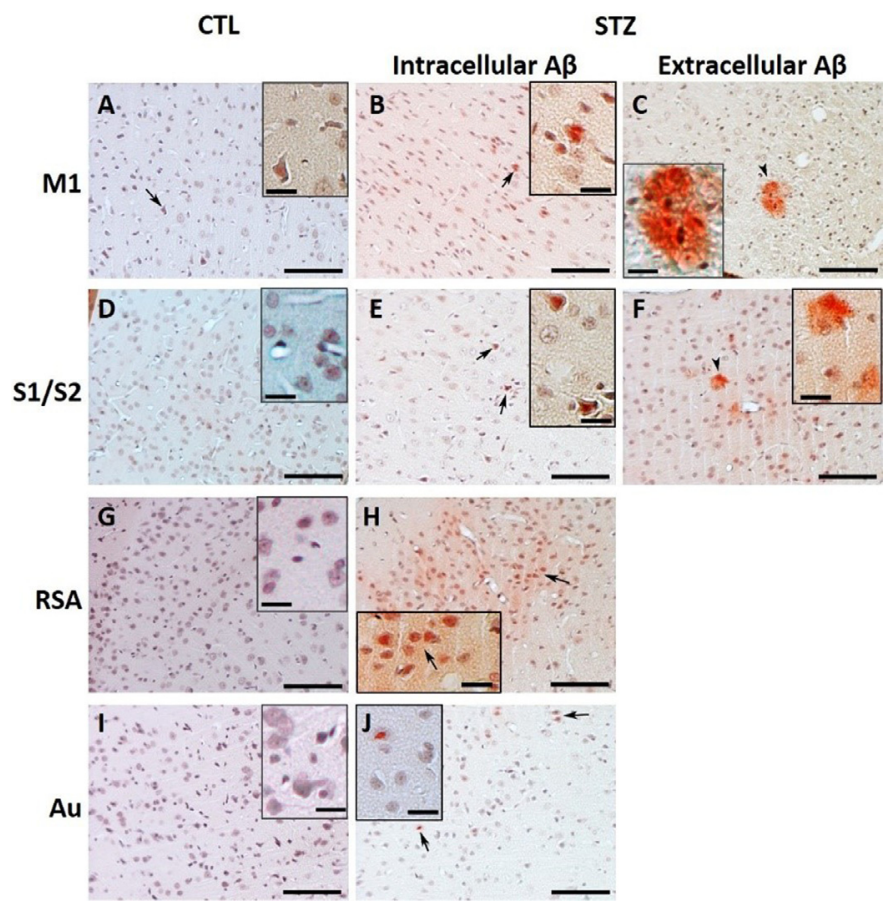

Fig. 8. $\mathrm{A} \beta$ accumulation in the brain visualized with Congo red histochemical stain. Representative microphotographs of the brain of STZ rats (columns 2 and 3) and age-matched controls (column 1). The STZ treatment revealed positive signal of intracellular (column 2, arrows) and extracellular (column 3, arrowheads) $\mathrm{A} \beta$ accumulation in the primary motor (M1), primary and secondary somatosensory (S1/S2), agranular retrosplenial (RSA) and auditory (Au) cortices. Scale bar: $100 \mu \mathrm{m}$ for main micrographs and $20 \mu \mathrm{m}$ for insets. (For interpretation of the references to color in this figure legend, the reader is referred to the online version of this article.)

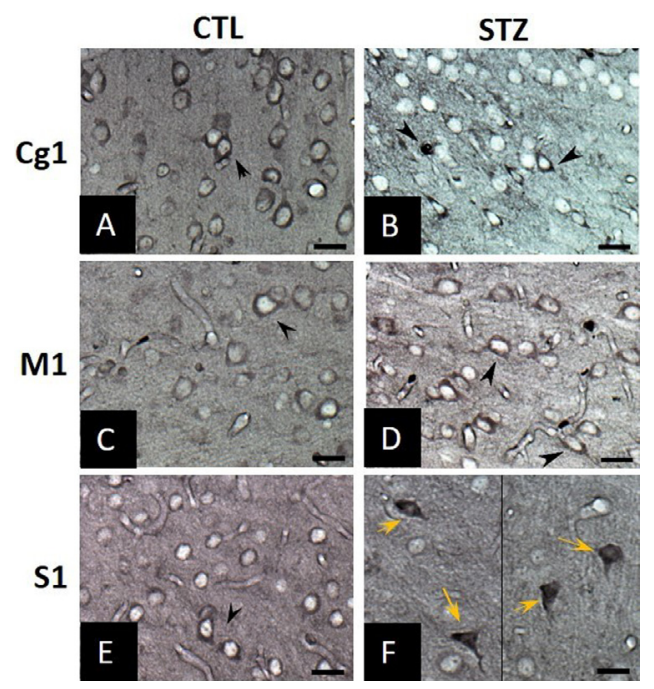

Fig. 9. Representative micrographs of Gallyas silver stain of STZ rats (B, D, F) and age-matched controls (A, C, E) revealed accumulation of abnormal tauprotein in the pre-tangle status (black arrowheads) in the cingulate cortex (Cg1), primary motor (M1) and somatosensory cortex (S1) in both groups. The STZ animals exhibited however more advanced forms of NFTs in S1 (F, yellow arrows). Scale bar: $20 \mu \mathrm{m}$. (For interpretation of the references to color in this figure legend, the reader is referred to the online version of this article.) 

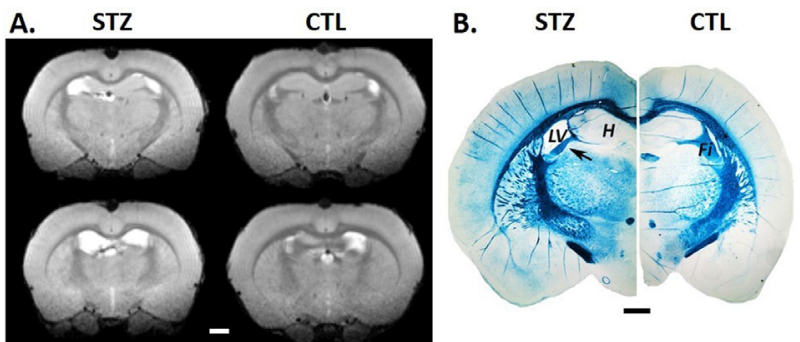

Fig. 10. Atrophy of the fimbria (Fi) and enlargement of lateral ventricle (LV) in STZ rats vs CTL at 21 weeks after icv injection. A) In vivo MRI anatomical image in two axial planes (anterior (top row) to posterior (bottom row) position). Scale bar: $2 \mathrm{~mm}$. B) LFB staining, confirming shrinkage of the fimbria (arrow) and significant enlargement of ventricular space (LV) in STZ rats. Scale bar $1 \mathrm{~mm}$. H: hippocampus.

coincided with altered axonal integrity and myelination of the cingulum bundle appearing at that timepoint. Temporally, the initial acute damage and partial recovery in white matter microstructure at 2 and 6 weeks, respectively, corresponds to an early phase of hyper-connectivity and impaired network dissociation, suggesting compensatory mechanisms, while the 13-week timepoint reveals widespread and pronounced white matter damage as well as loss of functional connectivity as markers of a chronic phase of disease.

Postmortem histology revealed pathological accumulation of amyloid in STZ rats in motor, somatosensory, retrosplenial and auditory cortices. Abnormal tau was also found in anterior cingulate, motor and somatosensory areas, though NFTs specific to the STZ group were found in S1 only and information from posterior parts of the brain was unfortunately missing. Furthermore, our histology measurements are only available at a later timepoint (21 weeks after icv injection). Histology reports at multiple timepoints on this animal model are available in the literature (Knezovic et al., 2015; Kraska et al., 2012; Shoham et al., 2003). While enlarged ventricles are not specific to AD (Apostolova et al., 2012), here we observed more substantial brain atrophy and enlarged ventricles in STZ animals as compared to controls in both the in vivo MRI data and postmortem histology, which were fully consistent with previous reports (Kraska et al., 2012; Shoham et al., 2003).

Our MRI findings of axonal integrity and functional connectivity were consistent with reported neuronal loss in the septum and corpus callosum (Kraska et al., 2012), thinning of the parietal cortex and the corpus callosum (Knezovic et al., 2015) and demyelination associated with microglia activation in the hippocampus and fimbria (Shoham et al., 2003). However, our histology analysis at 21 weeks found no amyloid in the hippocampus and this region could unfortunately not be evaluated for NFTs, while early neurofibrillary changes in the hippocampus have been reported at 12 weeks followed by presence of diffuse $\mathrm{A} \beta$ plaques at 26 weeks (Knezovic et al., 2015).

PET data showed reduced FDG uptake in icv-STZ rats in brain regions typically involved in $\mathrm{AD}$, and were consistent with reported changes in FDG uptake in the medial temporal lobe of icv-STZ monkeys as early as 2 weeks after injection (Heo et al., 2011). In our study, the most pronounced differences in uptake were found at 6 weeks, but the development of earlier glucose hypometabolism in STZ rats is highly likely. Indeed, the absence of SUVr group differences at the earliest timepoint was most probably due to a very limited PET cohort size (2 animals per group, Fig. 1), with STZ rats nonetheless displaying lower SUVr in PPC and MTL, qualitatively. Furthermore, STZ rats were on average already $70 \mathrm{~g}$ lighter than CTL rats at that timepoint (Fig. 3). The significant weight difference supports the assumption of impaired brain glucose metabolism as early as 2 weeks and the use of alternative energy sources such as stored body fat, which when burnt results in a build-up of ketones. Ketone bodies have been referred to as a "brain reserve" that can be mobilized to compensate for an inability to efficiently

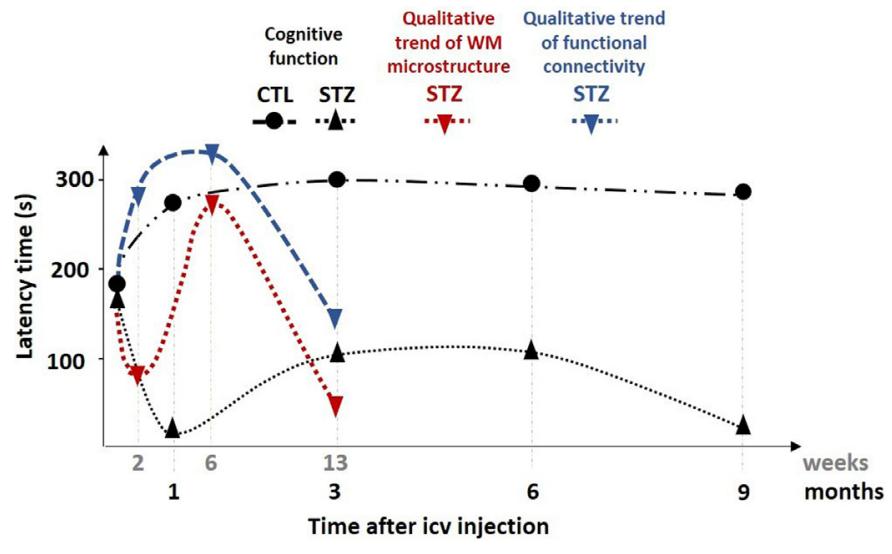

Fig. 11. Progression of MRI biomarkers in comparison with memory performance in the icv-STZ rat model. Figure adapted from (Knezovic et al., 2015). Black: Latency time measured in the passive avoidance test reported in (Knezovic et al., 2015) as a function of time in months. Red/blue: Qualitative trend of the diffusion/functional MRI biomarkers - respectively - reported in the present study as function of time in weeks. The plots of cognitive performance in CTL and STZ rats correspond to the follow-up of male Wistar rats that received an initial injection of either $3 \mathrm{mg} / \mathrm{kg}$ of STZ or buffer only, same as in our experiment. Differences between the procedures in Knezovic et al. and our experiments lie in the weight of rats at baseline ( $300 \mathrm{~g}$ vs $250 \mathrm{~g}$, respectively) and the fact that the STZ dose was split over 2 injections (day 1 and day 3) vs single injection, respectively. The timepoints of observation were also not identical but the dynamics are slower in the memory performance, with a chronic stage occurring around 26 weeks, while MRI-derived biomarkers point to an earlier switch towards pronounced neurodegeneration and reduced functional connectivity between 6 and 13 weeks.

utilize glucose (Gano et al., 2014; Wu et al., 2018). It is remarkable that early weight loss has also been reported in AD (Sergi et al., 2013; Tamura et al., 2008), although various explanations for it have been proposed, including a low leptin state associated with hypothalamic dysfunction (Ishii et al., 2014). Interestingly, STZ rats showed increased anti-correlations between the ACC and the hypothalamus connectivity at 13 weeks after injection. Along these lines, we propose that weight loss and/or slower weight gain in our STZ rats reflect: (1) a change in energy homeostasis; and (2) the effect of ketones on the signaling in the hypothalamus of appetite regulating neurohormones, such as leptin, that respond to changes in carbohydrate (glucose/glycogen) to fat macronutrient availability (Sumithran et al., 2013; Thio, 2012). Remarkably, we report "partial recovery" in terms of axonal integrity and functional hyperconnectivity at six weeks post-injection, in a context of marked FDG uptake differences at that timepoint. This observation follows the caveat that metabolism underlies structure and function, and supports the assumption that past the acute phase, the brain switches to other forms of energy, such as ketone bodies, as a means to maintain energy homeostasis and restore normal brain function (Yang et al., 2019). Interestingly, the same regions with reduced FDG uptake at 6 weeks after STZ injection eventually showed functional hypoconnectivity at 13 weeks (Fig. 4 and Fig. 5), supporting the manifestation of a chronic phase of disease. Here, our data suggests that neurodegeneration resumes as pathological mechanisms approach a critical stage and can no longer be compensated for.

The succession of acute change, compensation/partial remission and chronic degeneration phases, which exemplifies nonmonotonic changes, has also been reported in a nine-month follow-up of memory performance in icv-STZ rats, measured using a passive avoidance test (Knezovic et al., 2015). In this cognitive study however, the examination timepoints were different from ours and the acute phase was found at 1 month after injection, the recovery period between 3 and 6 months and chronic decrease of latency time from 6 months on. The curves of pro- 
gression of the MRI-derived biomarkers of neurodegeneration used in this study were qualitatively overlaid with that of memory performance from (Knezovic et al., 2015) for comparison (Fig. 11). The qualitative trends in white matter microstructure and functional connectivity provide potentially earlier evidence of brain damage in the icv-STZ model than behavioral observations, as MRI-derived biomarkers are expected to be more sensitive to mechanisms occurring in the preclinical stage of the pathological cascade. Such neuroimaging trends could potentially predict the following symptomatic stage of the disease that includes cognitive deficits.

Nonmonotonic and biphasic trajectories of brain alterations have also been reported in several human studies of $\mathrm{AD}$ and mild cognitive impairment, and involve a variety of biomarkers from cortical volumes, cerebral perfusion, gray and white matter microstructure, and hippocampal activity (Dickerson et al., 2005; Dong et al., 2020; Fortea et al., 2014, 2011; Montal et al., 2018; Pegueroles et al., 2017; Sierra-Marcos, 2017), which reinforces the suitability of the icv-STZ animal model for sporadic AD. Furthermore, the non-monotonic progression of AD in humans was reported based on cross-sectional studies including subjects at different stages of disease. The current study in icvSTZ rats highlights this nonmonotonic pattern in a longitudinal framework also, which is easier to achieve in animals than in humans. The acute phase identified using MRI-derived biomarkers could represent a valuable therapeutic window to strengthen the recovery and prevent or delay chronic degeneration, to be considered both in preclinical and clinical studies of AD.

The quantification of functional connectivity and axonal degeneration has provided insightful information about spatial and temporal AD-like changes induced by brain glucose hypometabolism. The present study contributes to the growing hypothesis that brain insulin resistance is a key player in the onset of $\mathrm{AD}$ and the icv-STZ rat model offers great potential for the investigation of neurodegeneration in the context of $\mathrm{AD}$ and diabetes. For a complete picture of the pathological cascade in this animal model, a future longitudinal study should include MRI, PET, behavior and cross-sectional histology all at once.

\section{Study limitations}

PET and fMRI experiments were conducted under different anesthetic conditions, which may complicate the comparison of brain activity between the two modalities, but not the between-group comparisons for each modality. Isoflurane induces a very stable and controlled anesthesia, which is well-suited for the safety requirements of PET experiments. The value of medetomidine vs isoflurane for rs-fMRI studies has however been largely shown in the literature (Kalthoff et al., 2013; Kint et al., 2020; Pawela et al., 2009), which is why this sedative was chosen for fMRI. Most recent literature suggests that a combination of medetomidine and low-dose isoflurane may be ideal for rat fMRI (Kint et al., 2020; Paasonen et al., 2018) and this is something that will be considered in future studies.

As $\mathrm{AD}$ is more prevalent in women than men, studies in animal models should ideally characterize both sexes to assess the full representativeness of the model. However, as hormonal modulation seems to play an important role in the vulnerability or resistance to $\mathrm{AD}$ progression, female rats should ideally be included at an advanced aged, as an equivalent of women's menopause. For example, a recent study has shown that hippocampal changes in icv-STZ rats were dependent on sex, remarkably with young female rats more resistant to STZ-induced alterations than males (Biasibetti et al., 2017). It is however not clear whether older females would not be more vulnerable to icv-STZ as a result of substantial hormonal changes. In our study, we used male rats only, which we acknowledge as a limitation. This choice was guided by practical reasons. However, we do plan to perform a similar study in aging female rats in the near future. More studies are indeed needed to better understand how gender differences and hormonal modulation can impact disease progression.

\section{Summary}

We used the icv-STZ rat model to characterize longitudinal alterations in brain functional connectivity and white matter integrity resulting from impaired brain glucose metabolism. Icv-STZ animals displayed early hyper-connectivity within the DMN and impaired network dissociation between the DMN and LCN followed by hypo-connectivity within the DMN. Moreover, white matter degeneration was manifested as intra-axonal damage and demyelination, potentially leading to axonal loss and mainly affecting the corpus callosum and the fimbria of the hippocampus. It followed a sequence of early acute injury followed by transient partial recovery and late chronic degeneration. Structural and functional changes presented matching spatio-temporal patterns, with a turning point between 6 and 13 weeks, and were in agreement with existing literature. Histology at a later timepoint confirmed pathological accumulation of amyloid and NFTs in STZ rats, in gray matter regions also impacted by altered functional connectivity. In parallel, brain glucose hypometabolism was likely established from the start. The initial hyper-connectivity and transient repair in brain structure at six weeks post-STZ injection is interpreted as the recruitment of alternative forms of energy such as ketone bodies to make up for impaired glucose metabolism, until pathological mechanisms are no longer reversible. Our results support the hypothesis of impaired glucose metabolism as a key player in the onset of $\mathrm{AD}$ and the relevance of the icv-STZ animal models for pre-clinical AD research. This study (i) characterized the impact of brain glucose hypometabolism on brain microstructure and function, (ii), highlighted signature nonmonotonic trajectories in their evolution and (iii) proposed potent MRI-derived biomarkers translatable to human $\mathrm{AD}$ and diabetic populations to monitor disease progression and potential response to treatment.

\section{Author contributions}

CTP: Software, Validation, Formal analysis, Investigation, Data Curation, Writing - Original draft, Visualization.

YD: Software, Validation, Formal analysis, Investigation, Writing Review \& Editing, Visualization.

TY: Software, Validation, Formal analysis, Writing - Review \& Editing.

ARdS: Investigation, Data Curation, Writing - Review \& Editing.

BL: Investigation, Formal analysis, Writing - Review \& Editing.

KP: Methodology, Investigation, Visualization, Writing - Review \& Editing.

CPY: Methodology, Investigation, Formal analysis, Writing - Review \& Editing.

IOJ: Conceptualization, Methodology, Software, Investigation, Writing - Original draft, Writing - Review \& Editing, Supervision, Project administration.

\section{Acknowledgements}

The authors thank Stefan Mitrea, Mario Lepore and Dario Sessa for technical assistance and the Histology Core Facility of the EPFL for staining procedures. This work was supported by the Center for Biomedical Imaging (CIBM) of the Vaud University Hospital (CHUV), the University of Lausanne (UNIL), the Swiss Federal Institute of Technology of Lausanne (EPFL), the University of Geneva (UniGe) and the Geneva University Hospital (HUG). C.T.P. acknowledges the support of the SwissEuropean Mobility Plan (Movetia).

\section{Supplementary materials}

Supplementary material associated with this article can be found, in the online version, at doi:10.1016/j.neuroimage.2020.117498. 


\section{References}

Ades-Aron, B., Veraart, J., Kochunov, P., McGuire, S., Sherman, P., Kellner, E., Novikov, D.S., Fieremans, E., 2018. Evaluation of the accuracy and precision of the diffusion parameter EStImation with Gibbs and NoisE removal pipeline. Neuroimage 183, 532-543. https://doi.org/10.1016/j.neuroimage.2018.07.066.

An, Y., Varma, V.R., Varma, S., Casanova, R., Dammer, E., Pletnikova, O., Chia, C.W., Egan, J.M., Ferrucci, L., Troncoso, J., Levey, A.I., Lah, J., Seyfried, N.T., Legido-Quigley, C., O'Brien, R., Thambisetty, M., 2018. Evidence for brain glucose dysregulation in Alzheimer's disease. Alzheimers Dement 14, 318-329. https://doi.org/10.1016/j.jalz.2017.09.011.

Andersson, J.L.R., Sotiropoulos, S.N., 2016. An integrated approach to correction for offresonance effects and subject movement in diffusion MR imaging. Neuroimage 125, 1063-1078. https://doi.org/10.1016/j.neuroimage.2015.10.019.

Apostolova, L.G., Green, A.E., Babakchanian, S., Hwang, K.S., Chou, Y.-.Y., Toga, A.W., Thompson, P.M., 2012. Hippocampal atrophy and ventricular enlargement in normal aging, mild cognitive impairment (MCI), and Alzheimer Disease. Alzheimer Dis. Assoc. Disord. 26, 17-27. https://doi.org/10.1097/WAD.0b013e3182163b62.

Attwell, D., Laughlin, S.B., 2001. An Energy Budget for Signaling in the Grey Matter of the Brain. J. Cereb. Blood Flow Metab. 21, 1133-1145. https://doi.org/10.1097/00004647-200110000-00001.

Avants, B., Epstein, C., Grossman, M., Gee, J., 2008. Symmetric diffeomorphic image registration with cross-correlation: evaluating automated labeling of elderly and neurodegenerative brain. Med. Image Anal. 12, 26-41. https://doi.org/10.1016/j.media.2007.06.004.

Avants, B., Tustison, N., Song, G., Cook, P.A., Klein, A., Gee, J.C., 2011. A reproducible evaluation of ANTs similarity metric performance in brain image registration. Neuroimage 54, 2033-2044. https://doi.org/10.1016/j.neuroimage.2010.09.025.

Balducci, C., Forloni, G., 2018. Novel targets in Alzheimer's disease: a special focus on microglia. Pharmacol. Res. 130, 402-413. https://doi.org/10.1016/j.phrs.2018.01.017.

Bartzokis, G., 2011. Alzheimer's disease as homeostatic responses to age-related myelin breakdown. Neurobiol Aging 32, 1341-1371. https://doi.org/10.1016/j.neurobiolaging.2009.08.007.

Bartzokis, G., 2004. Age-related myelin breakdown: a developmental model of cognitive decline and Alzheimer's disease. Neurobiol. Aging 25, 5-18. https://doi.org/10.1016/j.neurobiolaging.2003.03.001.

Beckmann, C.F., Smith, S.M., 2004. Probabilistic Independent Component Analysis for Functional Magnetic Resonance Imaging. IEEE Trans. Med. Imaging 23, 137-152. https://doi.org/10.1109/TMI.2003.822821

Benedikz, E., Kloskowska, E., Winblad, B., 2009. The rat as an animal model of Alzheimer's disease. J. Cell. Mol. Med. 13, 1034-1042. https://doi.org/10.1111/j.1582-4934.2009.00781.x.

Biasibetti, R., Almeida dos Santos, J.P., Rodrigues, L., Wartchow, K.M., Suardi, L.Z., Nardin, P., Selistre, N.G., Vázquez, D., Gonçalves, C.-.A., 2017. Hippocampal changes in STZ-model of Alzheimer's disease are dependent on sex. Behav. Brain Res. 316, 205-214. https://doi.org/10.1016/j.bbr.2016.08.057.

Binnewijzend, M.A.A., Schoonheim, M.M., Sanz-Arigita, E., Wink, A.M., van der Flier, W.M., Tolboom, N., Adriaanse, S.M., Damoiseaux, J.S., Scheltens, P., van Berckel, B.N.M., Barkhof, F., 2012. Resting-state fMRI changes in Alzheimer's disease and mild cognitive impairment. Neurobiol. Aging 33, 2018-2028. https://doi.org/10.1016/j.neurobiolaging.2011.07.003.

Bouhrara, M., Reiter, D.A., Bergeron, C.M., Zukley, L.M., Ferrucci, L., Resnick, S.M., Spencer, R.G., 2018. Evidence of demyelination in mild cognitive impairment and dementia using a direct and specific magnetic resonance imaging measure of myelin content. Alzheimers Dement 14, 998-1004. https://doi.org/10.1016/j.jalz.2018.03.007.

Brun, A., Englund, E., 1986. A white matter disorder in dementia of the Alzheimer type: a pathoanatomical study. Ann Neurol. 19, 253-262. https://doi.org/10.1002/ana.410190306.

Buxbaum, J.N., 2009. Animal models of human amyloidoses: are transgenic mice worth the time and trouble? FEBS Lett. 583, 2663-2673. https://doi.org/10.1016/j.febslet.2009.07.031.

Chang, Y.-.L., Chen, T.-.F., Shih, Y.-.C., Chiu, M.-.J., Yan, S.-.H., Tseng, W.-Y.I., 2015. Regional Cingulum Disruption, Not Gray Matter Atrophy, Detects Cognitive Changes in Amnestic Mild Cognitive Impairment Subtypes. J. Alzheimers Dis. 44, 125-138. https://doi.org/10.3233/JAD-141839.

Choo, I.H., Lee, D.Y., Oh, J.S., Lee, J.S., Lee, D.S., Song, I.C., Youn, J.C., Kim, S.G., Kim, K.W., Jhoo, J.H., Woo, J.I., 2010. Posterior cingulate cortex atrophy and regional cingulum disruption in mild cognitive impairment and Alzheimer's disease. Neurobiol. Aging 31, 772-779. https://doi.org/10.1016/j.neurobiolaging.2008.06.015.

Correia, S.C., Santos, R.X., Perry, G., Zhu, X., Moreira, P.I., Smith, M.A., 2011. Insulinresistant brain state: the culprit in sporadic Alzheimer's disease? Ageing Res. Rev. 10, 264-273. https://doi.org/10.1016/j.arr.2011.01.001

de la Monte, S.M., Wands, J.R., 2008. Alzheimer's disease is type 3 diabetes-evidence reviewed. J. Diab. Sci. Technol. 2, 1101-1113. https://doi.org/10.1177/193229680800200619.

Dhital, B., Reisert, M., Kellner, E., Kiselev, V.G., 2019. Intra-axonal diffusivity in brain white matter. Neuroimage 189, 543-550. https://doi.org/10.1016/j.neuroimage.2019.01.015

Diao, Y., Yin, T., Gruetter, R., Jelescu, I.O., 2020. An optimized pipeline for functional connectivity analysis in the rat brain. ArXiv Q-Bio 2001, 09857 https://arxiv.org/abs/2001.09857.

Dickerson, B., 2013. Biomarker-based prediction of progression in MCI: comparison of AD signature and hippocampal volume with spinal fluid amyloid- $\beta$ and tau. Front. Aging Neurosci. 5. https://doi.org/10.3389/fnagi.2013.00055.

Dickerson, B.C., Salat, D.H., Greve, D.N., Chua, E.F., Rand-Giovannetti, E., Rentz, D.M., Bertram, L., Mullin, K., Tanzi, R.E., Blacker, D., Albert, M.S.,
Sperling, R.A., 2005. Increased hippocampal activation in mild cognitive impairment compared to normal aging and AD. Neurology 65, 404. https://doi.org/10.1212/01.wnl.0000171450.97464.49.

Does, M.D., Olesen, J.L., Harkins, K.D., Serradas-Duarte, T., Gochberg, D.F., Jespersen, S.N., Shemesh, N., 2019. Evaluation of principal component analysis image denoising on multi-exponential MRI relaxometry. Magn. Reson. Med. 27658. https://doi.org/10.1002/mrm.27658.

Dong, J.W., Jelescu, I.O., Ades-Aron, B., Novikov, D.S., Friedman, K., Babb, J.S., Osorio, R.S., Galvin, J.E., Shepherd, T.M., Fieremans, E., 2020. Diffusion MRI biomarkers of white matter microstructure vary nonmonotonically with increasing cerebral amyloid deposition. Neurobiol. Aging 89, 118-128. https://doi.org/10.1016/j.neurobiolaging.2020.01.009.

Edison, P., Archer, H.A., Hinz, R., Hammers, A., Pavese, N., Tai, Y.F., Hotton, G., Cutler, D., Fox, N., Kennedy, A., Rossor, M., Brooks, D.J., 2007. Amyloid, hypometabolism, and cognition in Alzheimer disease: an [11C]PIB and [18F]FDG PET study. Neurology 68, 501-508. https://doi.org/10.1212/01.wnl.0000244749.20056.d4.

Englund, E., Brun, A., Alling, C., 1988. White matter changes in dementia of Alzheimer's type. Biochemical and neuropathological correlates. Brain J. Neurol. 111 (Pt 6), $1425-1439$.

Fieremans, E., Jensen, J.H., Helpern, J.A., 2011. White matter characterization with diffusional kurtosis imaging. Neuroimage 58, 177-188. https://doi.org/10.1016/j.neuroimage.2011.06.006.

Fortea, J., Sala-Llonch, R., Bartrés-Faz, D., Lladó, A., Solé-Padullés, C., Bosch, B., Antonell, A., Olives, J., Sanchez-Valle, R., Molinuevo, J.L., Rami, L., 2011. Cognitively preserved subjects with transitional cerebrospinal Fluid ß-Amyloid 1-42 values have thicker cortex in Alzheimer's disease vulnerable areas. Biol. Psychiatry 70, 183-190. https://doi.org/10.1016/j.biopsych.2011.02.017.

Fortea, J., Vilaplana, E., Alcolea, D., Carmona-Iragui, M., Sánchez-Saudinos, M.-.B., Sala, I., Antón-Aguirre, S., González, S., Medrano, S., Pegueroles, J., Morenas, E., Clarimón, J., Blesa, R., Lleó, A., for the Alzheimer's Disease Neuroimaging, I., 2014. Cerebrospinal fluid $\beta$-amyloid and phospho-tau biomarker interactions affecting brain structure in preclinical Alzheimer disease. Ann. Neurol 76, 223-230. https://doi.org/10.1002/ana.24186

Fox, M.D., Snyder, A.Z., Vincent, J.L., Corbetta, M., Van Essen, D.C., Raichle, M.E., 2005. The human brain is intrinsically organized into dynamic, anticorrelated functional networks. Proc. Natl. Acad. Sci. 102, 9673-9678. https://doi.org/10.1073/pnas.0504136102.

Franzmeier, N., Ren, J., Damm, A., Monté-Rubio, G., Boada, M., Ruiz, A., Ramirez, A. Jessen, F., Düzel, E., Rodríguez Gómez, O., Benzinger, T., Goate, A., Karch, C.M., Fagan, A.M., McDade, E., Buerger, K., Levin, J., Duering, M., Dichgans, M., SuárezCalvet, M., Haass, C., Gordon, B.A., Lim, Y.Y., Masters, C.L., Janowitz, D., Catak, C., Wolfsgruber, S., Wagner, M., Milz, E., Moreno-Grau, S., Teipel, S., Grothe, M.J., Kilimann, I., Rossor, M., Fox, N., Laske, C., Chhatwal, J., Falkai, P., Perneczky, R., Lee, J..H., Spottke, A., Boecker, H., Brosseron, F., Fliessbach, K., Heneka, M.T., Nestor, P., Peters, O., Fuentes, M., Menne, F., Priller, J., Spruth, E.J., Franke, C., Schneider, A., Westerteicher, C., Speck, O., Wiltfang, J., Bartels, C., Araque Caballero, M.Á., Metzger, C., Bittner, D., Salloway, S., Danek, A., Hassenstab, J., Yakushev, I., Schofield, P.R., Morris, J.C., Bateman, R.J., Ewers, M., 2019. The BDNFVal66Met SNP modulates the association between beta-amyloid and hippocampal disconnection in Alzheimer's disease. Mol. Psychiatry. https://doi.org/10.1038/s41380-019-0404-6.

Friston, K., Ashburner, J., Kiebel, S., Nichols, T., Penny, W., 2007. Statistical Parametric Mapping. Elsevier https://doi.org/10.1016/B978-0-12-372560-8.X5000-1.

Ganda, O.P., Rossini, A.A., Like, A.A., 1976. Studies on streptozotocin diabetes. Diabetes 25, 595-603.

Gano, L.B., Patel, M., Rho, J.M., 2014. Ketogenic diets, mitochondria, and neurological diseases. J. Lipid Res. 55, 2211-2228. https://doi.org/10.1194/jlr.R048975.

Gispert, J.D., Rami, L., Sánchez-Benavides, G., Falcon, C., Tucholka, A., Rojas, S., Molinuevo, J.L., 2015. Nonlinear cerebral atrophy patterns across the Alzheimer's disease continuum: impact of APOE4 genotype. Neurobiol. Aging 36, 2687-2701. https://doi.org/10.1016/j.neurobiolaging.2015.06.027.

Gozzi, A., Schwarz, A.J., 2016. Large-scale functional connectivity networks in the rodent brain. Neuroimage 127, 496-509. https://doi.org/10.1016/j.neuroimage.2015.12.017.

Griffanti, L., Douaud, G., Bijsterbosch, J., Evangelisti, S., Alfaro-Almagro, F., Glasser, M.F., Duff, E.P., Fitzgibbon, S., Westphal, R., Carone, D., Beckmann, C.F., Smith, S.M., 2017. Hand classification of fMRI ICA noise components. Neuroimage 154, 188-205. https://doi.org/10.1016/j.neuroimage.2016.12.036.

Gudala, K., Bansal, D., Schifano, F., Bhansali, A., 2013. Diabetes mellitus and risk of dementia: a meta-analysis of prospective observational studies. J. Diabetes Investig. 4, 640-650. https://doi.org/10.1111/jdi.12087.

Heo, J.-.H., Lee, S.-.R., Lee, S.-.T., Lee, K.-.M., Oh, J.-.H., Jang, D.-.P., Chang, K.-.T., Cho, Z.-.H., 2011. Spatial distribution of glucose hypometabolism induced by intracerebroventricular streptozotocin in monkeys. J. Alzheimers Dis. 25, 517-523. https://doi.org/10.3233/JAD-2011-102079.

Hölscher, C., 2019. Insulin signaling impairment in the brain as a risk factor in Alzheimer's disease. Front. Aging Neurosci. 11, 88. https://doi.org/10.3389/fnagi.2019.00088.

Ishii, M., Wang, G., Racchumi, G., Dyke, J.P., Iadecola, C., 2014. Transgenic mice overexpressing amyloid precursor protein exhibit early metabolic deficits and a pathologically low leptin state associated with hypothalamic dysfunction in arcuate neuropeptide Y neurons. J. Neurosci. 34, 9096-9106. https://doi.org/10.1523/JNEUROSCI.0872-14.2014.

Jack, C.R., Knopman, D.S., Jagust, W.J., Petersen, R.C., Weiner, M.W., Aisen, P.S., Shaw, L.M., Vemuri, P., Wiste, H.J., Weigand, S.D., Lesnick, T.G., Pankratz, V.S., Donohue, M.C., Trojanowski, J.Q., 2013. Tracking pathophysiological processes in Alzheimer's disease: an updated hypothetical model of dynamic biomarkers. Lancet Neurol. 12, 207-216. https://doi.org/10.1016/S1474-4422(12)70291-0. 
Jelescu, I.O., Budde, M.D., 2017. Design and validation of diffusion MRI models of white matter. Front. Phys. 5, 61. https://doi.org/10.3389/fphy.2017.00061

Jelescu, I.O., Palombo, M., Bagnato, F., Schilling, K.G., 2020. Challenges for biophysical modeling of microstructure. Journal of Neuroscience Methods 344, 108861. doi:10.1016/j.jneumeth.2020.108861.

Jelescu, I.O., Shepherd, T.M., Novikov, D.S., Ding, Y.-.S., Ades-Aron, B., Smith, J., Vahle, T., Babb, J.S., Friedman, K.P., de Leon, M.J., Golomb, J.B., Galvin, J.E., Fieremans, E., 2018. Spatial relationships between white matter degeneration, amyloid load and cortical volume in amnestic mild cognitive impairment. bioRxiv 441840 . https://doi.org/10.1101/441840.

Jelescu, I.O., Veraart, J., Fieremans, E., Novikov, D.S., 2016a. Degeneracy in model parameter estimation for multi-compartmental diffusion in neuronal tissue. NMR Biomed. 29, 33-47. https://doi.org/10.1002/nbm.3450.

Jelescu, I.O., Zurek, M., Winters, K.V., Veraart, J., Rajaratnam, A., Kim, N.S., Babb, J.S., Shepherd, T.M., Novikov, D.S., Kim, S.G., Fieremans, E., 2016b. In vivo quantification of demyelination and recovery using compartment-specific diffusion MRI metrics validated by electron microscopy. Neuroimage 132, 104-114. https://doi.org/10.1016/j.neuroimage.2016.02.004

Jenkinson, M., Bannister, P., Brady, M., Smith, S., 2002. Improved optimization for the robust and accurate linear registration and motion correction of brain images. Neuroimage 17, 825-841. https://doi.org/10.1006/nimg.2002.1132.

Jensen, J.H., Helpern, J.A., Ramani, A., Lu, H., Kaczynski, K., 2005. Diffusional kurtosis imaging: the quantification of non-gaussian water diffusion by means of magnetic resonance imaging. Magn. Reson. Med. 53, 1432-1440. https://doi.org/10.1002/mrm.20508.

Jespersen, S.N., Olesen, J.L., Hansen, B., Shemesh, N., 2018. Diffusion time dependence of microstructural parameters in fixed spinal cord. Neuroimage 182, 329-342. https://doi.org/10.1016/j.neuroimage.2017.08.039.

Junod, A., Lambert, A.E., Stauffacher, W., Renold, A.E., 1969. Diabetogenic action of streptozotocin: relationship of dose to metabolic response. J. Clin. Invest. 48, 2129-2139. https://doi.org/10.1172/jci106180.

Kalthoff, D., Po, C., Wiedermann, D., Hoehn, M., 2013. Reliability and spatial specificity of rat brain sensorimotor functional connectivity networks are superior under sedation compared with general anesthesia: RSFMRI under sedation and anesthesia. NMR Biomed. 26, 638-650. https://doi.org/10.1002/nbm.2908.

Kametani, F., Hasegawa, M., 2018. Reconsideration of amyloid hypothesis and tau hypothesis in Alzheimer's Disease. Front. Neurosci. 12, 25. https://doi.org/10.3389/fnins.2018.00025.

Karthick, C., Periyasamy, S., Jayachandran, K., Anusuyadevi, M., 2016. Intrahippocampal administration of ibotenic acid induced cholinergic dysfunction via NR2A/NR2B expression: implications of resveratrol against Alzheimer disease pathophysiology. Front. Mol. Neurosci.. https://doi.org/10.3389/fnmol.2016.00028.

Kellner, E., Dhital, B., Kiselev, V.G., Reisert, M., 2016. Gibbs-ringing artifact removal based on local subvoxel-shifts: gibbs-Ringing Artifact Removal. Magn. Reson. Med. 76, 1574-1581. https://doi.org/10.1002/mrm.26054.

King, A., 2018. The search for better animal models of Alzheimer's disease. Nature 559, S13-S15. https://doi.org/10.1038/d41586-018-05722-9.

Kint, L., Seewoo, B., Hyndman, T., Clarke, M., Edwards, S., Rodger, J., Feindel, K., Musk, G., 2020. The pharmacokinetics of medetomidine administered subcutaneously during isoflurane anaesthesia in sprague-dawley rats. Animals 106, 1050. https://doi.org/10.3390/ani10061050.

Knezovic, A., Osmanovic-Barilar, J., Curlin, M., Hof, P.R., Simic, G., Riederer, P., SalkovicPetrisic, M., 2015. Staging of cognitive deficits and neuropathological and ultrastructural changes in streptozotocin-induced rat model of Alzheimer's disease. J. Neural Transm. 122, 577-592. https://doi.org/10.1007/s00702-015-1394-4.

Kobayashi, K., Hayashi, M., Nakano, H., Fukutani, Y., Sasaki, K., Shimazaki, M. Koshino, Y., 2002. Apoptosis of astrocytes with enhanced lysosomal activity and oligodendrocytes in white matter lesions in Alzheimer's disease. Neuropathol. Appl. Neurobiol. 28, 238-251.

Kraska, A., Santin, M.D., Dorieux, O., Joseph-Mathurin, N., Bourrin, E., Petit, F., Jan, C., Chaigneau, M., Hantraye, P., Lestage, P., Dhenain, M., 2012. In vivo cross-sectional characterization of cerebral alterations induced by in tracerebroventricular administration of streptozotocin. PLOS ONE 7, e46196. https://doi.org/10.1371/journal.pone.0046196.

Kuehn, B.M., 2020. In Alzheimer research, glucose metabolism moves to center stage. JAMA 323 (4), 297-299. https://doi.org/10.1001/jama.2019.20939.

Kunz, N., da Silva, A.R., Jelescu, I.O., 2018. Intra- and extra-axonal axial diffusivities in the white matter: which one is faster? Neuroimage 181, 314-322. https://doi.org/10.1016/j.neuroimage.2018.07.020.

Lanz, B., Poitry-Yamate, C., Gruetter, R., 2014. Image-derived input function from the vena cava for 18F-FDG PET studies in rats and mice. J. Nucl. Med. 55, 1380-1388. https://doi.org/10.2967/jnumed.113.127381.

Lester-Coll, N., Rivera, E.J., Soscia, S.J., Doiron, K., Wands, J.R., de la Monte, S.M., 2006. Intracerebral streptozotocin model of type 3 diabetes: relevance to sporadic Alzheimer's disease. J. Alzheimers Dis. 9, 13-33. doi:10.3233/JAD-2006-9102.

Montal, V., Vilaplana, E., Alcolea, D., Pegueroles, J., Pasternak, O., González-Ortiz, S., Clarimón, J., Carmona-Iragui, M. Illán-Gala, I., Morenas-Rodríguez, E., RibosaNogué, R., Sala, I., Sánchez-Saudinós, M.-.B., García-Sebastian, M., Villanúa, J., Izagirre, A., Estanga, A., Ecay-Torres, M., Iriondo, A., Clerigue, M., Tainta, M., Pozueta, A., González, A., Martínez-Heras, E., Llufriu, S., Blesa, R., Sanchez-Juan, P., Martínez-Lage, P., Lleó, A., Fortea, J., 2018. Cortical microstructural changes along the Alzheimer's disease continuum. Alzheimers Dement. J. Alzheimers Assoc. 14, 340351. https://doi.org/10.1016/j.jalz.2017.09.013.

Nitsch, R., Hoyer, S., 1991. Local action of the diabetogenic drug, streptozotocin, on glucose and energy metabolism in rat brain cortex. Neurosci. Lett. 128, 199-202. https://doi.org/10.1016/0304-3940(91)90260-Z.
Novikov, D.S., Veraart, J., Jelescu, I.O., Fieremans, E., 2018. Rotationally-invariant mapping of scalar and orientational metrics of neuronal microstructure with diffusion MRI Neuroimage 174, 518-538. https://doi.org/10.1016/j.neuroimage.2018.03.006.

Paasonen, J., Stenroos, P., Salo, R.A., Kiviniemi, V., Gröhn, O., 2018. Functional connectivity under six anesthesia protocols and the awake condition in rat brain. Neuroimage 172, 9-20. https://doi.org/10.1016/j.neuroimage.2018.01.014.

Pawela, C.P., Biswal, B.B., Hudetz, A.G., Schulte, M.L., Li, R., Jones, S.R., Cho, Y.R., Matloub, H.S., Hyde, J.S., 2009. A protocol for use of medetomidine anesthesia in rats for extended studies using task-induced BOLD contrast and resting-state functional connectivity. Neuroimage 46, 1137-1147. https://doi.org/10.1016/j.neuroimage.2009.03.004.

Pegueroles, J., Vilaplana, E., Montal, V., Sampedro, F., Alcolea, D., Carmona-Iragui, M., Clarimon, J., Blesa, R., Lleó, A., Fortea, J., 2017. Longitudinal brain structural changes in preclinical Alzheimer's disease. Alzheimers Dement 13, 499-509. https://doi.org/10.1016/j.jalz.2016.08.010.

Plaschke, K., Kopitz, J., Siegelin, M., Schliebs, R., Salkovic-Petrisic, M., Riederer, P., Hoyer, S., 2010. Insulin-resistant brain state after intracerebroventricular streptozotocin injection exacerbates Alzheimer-like changes in Tg2576 A $\beta$ PP-overexpressing mice. J. Alzheimers Dis. 19, 691-704. https://doi.org/10.3233/JAD-2010-1270.

Reynaud, O., da Silva, A.R., Gruetter, R., Jelescu, I.O., 2019. Multi-slice passband bSSFP for human and rodent fMRI at ultra-high field. J. Magn. Reson. 305, 31-40. https://doi.org/10.1016/j.jmr.2019.05.010.

Roher, A.E., Weiss, N., Kokjohn, T.A., Kuo, Y.M., Kalback, W., Anthony, J., Watson, D., Luehrs, D.C., Sue, L., Walker, D., Emmerling, M., Goux, W., Beach, T., 2002. Increased A beta peptides and reduced cholesterol and myelin proteins characterize white matter degeneration in Alzheimer's disease. Biochemistry 41, 11080-11090.

Sanganahalli, B.G., Herman, P., Behar, K.L., Blumenfeld, H., Rothman, D.L., Hyder, F., 2013. Functional MRI and neural responses in a rat model of Alzheimer's disease. Neuroimage 79, 404-411. https://doi.org/10.1016/j.neuroimage.2013.04.099.

Sergi, G., Rui, M.D., Coin, A., Inelmen, E.M., Manzato, E., 2013. Weight loss and Alzheimer's disease: temporal and aetiologic connections. Proc. Nutr. Soc. 72, 160 165. https://doi.org/10.1017/S0029665112002753.

Shankar, G.M., Walsh, D.M., 2009. Alzheimer's disease: synaptic dysfunction and A $\beta$. Mol. Neurodegener 4, 48. https://doi.org/10.1186/1750-1326-4-48.

Shaw, L.M., Vanderstichele, H., Knapik-Czajka, M., Clark, C.M., Aisen, P.S., Petersen, R.C., Blennow, K., Soares, H., Simon, A., Lewczuk, P., Dean, R., Siemers, E., Potter, W. Lee, V.M.-Y., Trojanowski, J.Q., Initiative, Alzheimer's Disease Neuroimaging, 2009. Cerebrospinal fluid biomarker signature in Alzheimer's disease neuroimaging initiative subjects. Ann. Neurol. 65, 403-413. https://doi.org/10.1002/ana.21610.

Shoham, S., Bejar, C., Kovalev, E., Weinstock, M., 2003. Intracerebroventricular injection of streptozotocin causes neurotoxicity to myelin that contributes to spatial memory deficits in rats. Exp. Neurol. 184, 1043-1052. https://doi.org/10.1016/j.expneurol.2003.08.015.

Sierra-Marcos, A., 2017. Regional cerebral blood flow in mild cognitive impairment and Alzheimer's disease measured with arterial spin labeling magnetic resonance imaging. Int. J. Alzheimers Dis. 2017. 5479597-5479597 https://doi.org/10.1155/2017/5479597

Smith, S.M., Jenkinson, M., Woolrich, M.W., Beckmann, C.F., Behrens, T.E.J., JohansenBerg, H., Bannister, P.R., De Luca, M., Drobnjak, I., Flitney, D.E., Niazy, R.K., Saunders, J., Vickers, J., Zhang, Y., De Stefano, N., Brady, J.M., Matthews, P.M., 2004. Advances in functional and structural MR image analysis and implementation as FSL. Neuroimage 23, S208-S219. https://doi.org/10.1016/j.neuroimage.2004.07.051.

Sperling, R.A., Aisen, P.S., Beckett, L.A., Bennett, D.A., Craft, S., Fagan, A.M., Iwatsubo, T., Jack, C.R., Kaye, J., Montine, T.J., Park, D.C., Reiman, E.M., Rowe, C.C., Siemers, E., Stern, Y., Yaffe, K., Carrillo, M.C., Thies, B., Morrison-Bogorad, M., Wagster, M.V., Phelps, C.H., 2011. Toward defining the preclinical stages of Alzheimer's disease: recommendations from the National Institute on Aging-Alzheimer's Association workgroups on diagnostic guidelines for Alzheimer's disease. Alzheimers Dement 7, 280 292. https://doi.org/10.1016/j.jalz.2011.03.003.

Stefanova, N.A., Maksimova, K.Y., Kiseleva, E., Rudnitskaya, E.A., Muraleva, N.A., Kolosova, N.G., 2015. Melatonin attenuates impairments of structural hippocampal neuroplasticity in OXYS rats during active progression of Alzheimer's disease-like pathology. J. Pineal Res. 59, 163-177. https://doi.org/10.1111/jpi.12248.

Sumithran, P., Prendergast, L.A., Delbridge, E., Purcell, K., Shulkes, A., Kriketos, A., Proietto, J., 2013. Ketosis and appetite-mediating nutrients and hormones after weight loss. Eur. J. Clin. Nutr. 67, 759-764. https://doi.org/10.1038/ejcn.2013.90.

Svennerholm, L., Gottfries, C.G., 1994. Membrane lipids, selectively diminished in Alzheimer brains, suggest synapse loss as a primary event in early-onset form (type I) and demyelination in late-onset form (type II). J. Neurochem. 62, 1039-1047.

Tamura, B.K., Masaki, K.H., Blanchette, P.L., 2008. Weight loss in patients with Alzheimer's Disease. J. Nutr. Elder. 26, 21-38. https://doi.org/10.1300/J052v26n03_02.

Thio, L.L., 2012. Hypothalamic hormones and metabolism. Epilepsy Res., Special Issue Dietary Treatments Epilepsy Neurolog. Disord. 100, 245-251. https://doi.org/10.1016/j.eplepsyres.2011.07.009.

van de Looij, Y., Kunz, N., Huppi, P., Gruetter, R., Sizonenko, S., 2011. Diffusion tensor echo planar imaging using surface coil transceiver with a semiadiabatic RF pulse sequence at 14.1T. Magn. Reson. Med. 65, 732-737. https://doi.org/10.1002/mrm.22656.

Veraart, J., Novikov, D.S., Christiaens, D., Ades-aron, B., Sijbers, J., Fieremans, E., 2016. Denoising of diffusion MRI using random matrix theory. Neuroimage 142, 394-406. https://doi.org/10.1016/j.neuroimage.2016.08.016.

Veraart, J., Novikov, D.S., Fieremans, E., 2018. TE dependent diffusion imaging (TEdDI) distinguishes between compartmental T2 relaxation times. Neuroimage 182, 360369. https://doi.org/10.1016/j.neuroimage.2017.09.030. 
Veraart, J., Sijbers, J., Sunaert, S., Leemans, A., Jeurissen, B., 2013. Weighted linear least squares estimation of diffusion MRI parameters: strengths, limitations, and pitfalls. Neuroimage 81, 335-346. https://doi.org/10.1016/j.neuroimage.2013.05.028.

Wang, L., Brier, M.R., Snyder, A.Z., Thomas, J.B., Fagan, A.M., Xiong, C., Benzinger, T.L., Holtzman, D.M., Morris, J.C., Ances, B.M., 2013. Cerebrospinal fluid A $\beta 42$, phosphorylated Tau ${ }_{181}$, and resting-state functional connectivity. JAMA Neurology 70 (10), 1242-1248. https://doi.org/10.1001/jamaneurol.2013.3253.

Weber, R., Ramos-Cabrer, P., Wiedermann, D., van Camp, N., Hoehn, M., 2006. A fully noninvasive and robust experimental protocol for longitudinal fMRI studies in the rat. Neuroimage 29, 1303-1310. https://doi.org/10.1016/j.neuroimage.2005.08.028.

Wu, L., Zhang, X., Zhao, L., 2018. Human ApoE isoforms differentially modulate brain glucose and ketone body metabolism: implications for Alzheimer's disease risk reduction and early intervention. J. Neurosci. 38, 6665-6681. https://doi.org/10.1523/JNEUROSCI.2262-17.2018.
Yang, H., Shan, W., Zhu, F., Wu, J., Wang, Q., 2019. Ketone bodies in neurological diseases: focus on neuroprotection and underlying mechanisms. Front. Neurol. 10 https://doi.org/10.3389/fneur.2019.00585.

Zarow, C., Vinters, H.V., Ellis, W.G., Weiner, M.W., Mungas, D., White, L., Chui, H.C. 2005. Correlates of hippocampal neuron number in Alzheimer's disease and ischemic vascular dementia. Ann. Neurol. 57, 896-903. https://doi.org/10.1002/ana.20503.

Zerbi, V., Grandjean, J., Rudin, M., Wenderoth, N., 2015. Mapping the mouse brain with rs-fMRI: an optimized pipeline for functional network identification. Neuroimage 123, 11-21. https://doi.org/10.1016/j.neuroimage.2015.07.090. 